\title{
Modeling the X-ray Process, and X-ray Flaw Size Parameter for POD Studies
}

\author{
Ajay M. Koshti, NASA Johnson Space Center
}

\begin{abstract}
Nondestructive evaluation (NDE) method reliability can be determined by a statistical flaw detection study called probability of detection (POD) study. In many instances, the NDE flaw detectability is given as a flaw size such as crack length. The flaw is either a crack or behaving like a crack in terms of affecting the structural integrity of the material. An alternate approach is to use a more complex flaw size parameter. The X-ray flaw size parameter, given here, takes into account many setup and geometric factors. The flaw size parameter relates to X-ray image contrast and is intended to have a monotonic correlation with the POD. Some factors such as set-up parameters, including X-ray energy, exposure, detector sensitivity, and material type that are not accounted for in the flaw size parameter may be accounted for in the technique calibration and controlled to meet certain quality requirements. The proposed flaw size parameter and the computer application described here give an alternate approach to conduct the POD studies. Results of the POD study can be applied to reliably detect small flaws through better assessment of effect of interaction between various geometric parameters on the flaw detectability. Moreover, a contrast simulation algorithm for a simple part-source-detector geometry using calibration data is also provided for the POD estimation.
\end{abstract}

Keywords: X-ray, flaw size parameter

\section{INTRODUCTION}

The paper provides modeling of X-ray flaw imaging contrast that is necessary to define the X-ray flaw size parameters. Commercial X-ray simulation software ${ }^{1,2}$ also model X-ray flaw detection and provide simulated images. The approach given here does not provide simulated X-ray images. Currently the commercial simulation software does not provide calculation of an X-ray flaw size parameter. However, similar to the approach given here, commercial X-ray simulation software can also be used to perform POD $^{3}$ studies. Comparison of results with commercial X-ray simulation software and its use in POD studies is not covered in this paper. The model based X-ray parameter and simulated contrast are intended for use in POD studies and the approach can be referred to as a model assisted POD. The paper however does not provide any information on the POD analysis and limits to defining input quantities that are needed in the POD analysis process.

\section{NDE FLAW DETECTABILITY SIZE}

Crack location, crack orientation as well as crack opening influence the NDE flaw detectability. Flaw detectability is affected by choice of the nondestructive method, equipment capability e.g. sensitivity and resolution; and set-up parameters. Generally, a physical standard is used to calibrate and verify sensitivity and resolution requirements. Once performance of an NDE procedure in terms of the expected minimum results on a relevant physical standard is established, the procedure can be implemented for detection of real flaws. Flaw detectability of an NDE method can be given in terms of a reliably detectable flaw size. This is typically given as a flaw size with $90 \%$ probability of detection with $95 \%$ confidence and is denoted as $a 90 / 95^{3}$. A POD demonstration study is conducted where a set of flaws within the desired range of sizes is chosen for detection using a documented and controlled NDE procedure. The $a 90 / 95$ flaw size is calculated by analyzing the demonstration results using a POD analysis software. It is important to remember that these estimations are dependent on number of flaws used in the demonstration. As the number of flaws in the POD demonstration increases, the $a 90 / 95$ estimate tends to approach the true $a 90$ value. It is customary to choose a definition of the flaw size parameter such that the POD increases with the flaw size parameter. Thus, the $a 90 / 95$ flaw size is normally larger than the true $a 90$ which is unknown. In some situations, especially where limited data is available, the a90/95 may not be assessed but instead a conservative value that is larger than the $a 90$ is calculated. Crack depth-to-partthickness ratio, $a / t$ is commonly used parameter for estimation of $a 90 / 95$ in X-ray inspection of metals ${ }^{4}$. A monotonically increasing signal response is expected with increasing flaw size parameter value within a desired range of the parameter. 


\section{X-RAY FLAW DETECTION ASSESSMENT APPROACH}

Many factors affect X-ray crack detectability. X-ray technique related factors are X-ray angle with respect to the crack plane, geometric unsharpness, detector plane angle, detector-part-source geometry, X-ray energy, exposure, detector type, detector sensitivity, detector noise, detector resolution, X-ray filter, and single wall versus double wall part geometry etc. X-ray technique quality indicators (e.g. film density, $2 \%$ penetrameter hole detectability) are used to verify technique sensitivity and resolution. One of the factors, X-ray scatter, is dependent upon the energy of X-rays, part material and geometry. Moreover, the detector may have an effect on the X-ray scatter appearing in the image. The effect of X-ray scatter is not addressed in this work. The X-ray scatter and its effect is assumed to be low, uniform, and controlled. The scatter is considered to be one of the factors contributing to noise in the data. Crack, material and part related factors are: crack depth, crack opening, crack angle, crack location, flatness and roughness of the crack faces, crack length; location of crack (e.g. in weld or parent material, surface roughness in the area of crack) and conditions that affect spatial noise (e.g. grain structure, scattering, part and weld geometry). Although, from fracture mechanics analysis point of view, it is desirable to have the NDE flaw size parameter to be only related to the crack major dimensions (e.g. length and depth), from NDE point of view, it is necessary to correlate flaw detection capability to both crack and key technique parameters. Currently, a90/95 crack depth-to-part thickness ratio (e.g. a/t) for film radiography in metal alloys is given as $70 \%$ for Standard NDE requirements ${ }^{4}$. The $70 \%$ number is arrived at by performing an X-ray flaw detectability study that meets the standard film X-ray requirements.

We intend to build some models that relate selected factors such as the surface crack dimensions, orientation, part geometry, and technique parameters to film or image contrast for a simulated crack in a plate. Although the detector sensitivity, noise, X-ray exposure and X-ray attenuation coefficient affect flaw detectability, we assume that we could capture these factors in detector response calibration curves and as technique requirements. This allows us to focus on the geometric factors of flaw and source-part-detector geometry. A geometric flaw size parameter is intended to be monotonically related to the POD under these conditions. This approach assumes that the flaw size parameter and technique requirements including the part material requirements, the detector response calibration characteristics together provide a complete description of the X-ray flaw detection application and provide sufficient information for assessment of the NDE capability, i.e. whether the flaw size under consideration can be reliably detected. A simulated contrast model provided here can do the same assessment if correlations are established between the simulated contrast and the actual contrast; and the simulation contrast and the POD. Applicability of these approaches would depend on validity of assumptions which affect accuracy of the models used. Therefore, experimental correlation with the signal response or POD is necessary to validate the models.

\section{OUTLINE OF THE MODEL DEVELOPMENT}

The paper is fairly lengthy in terms of number of equations. Therefore, some explanation of steps taken to build the Xray parameter and contrast model are necessary. Many quantities and some modeling approaches must be defined before the X-ray parameter and contrast can be defined. Initially, basic definitions and descriptions of the crack shape, geometry of the set-up; X-ray attenuation, X-ray intensity, exposure, film density, image intensity, and contrast are given. The definitions are followed by normalized detector response from a step wedge. The detector can be a film or a digital detector. Linear and non-linear detector responses are provided as a function of ratio $(a / t)$ of a step wedge. The models include a noise term. Definitions of the contrast and normalized contrast are given. These definitions and models are necessary for development of the X-ray parameter in later sections. Definitions of relative noise and contrast-to-noise ratio are given as these quantities are useful in the POD study. In order to model a detector, a definition and model of the modulation transfer function are given. The detector contrast and normalized contrast that account for effect of the modulation transfer function are provided as a function of the X-ray parameter. Next, three models of the X-ray parameter are developed. Finally, a computer application is developed to calculate the X-ray parameters and simulated contrasts. Explanation of use of these quantities in the X-ray NDE POD study is provided. The paper, however, does not provide any experimental data in the use of these quantities in a POD study.

Fig. 1 provides four separate blocks of the model development and general progression of the model development between these blocks. The first block lists basic quantities that have been defined. These quantities are necessary in the development of model. The second block provides a flow chart for modeling of the X-ray parameter. The third block provides a flow chart for modeling of the detector response. The fourth block provides a flow chart of modeling of MTF and detector response accounted X-ray parameter and simulated contrast. 


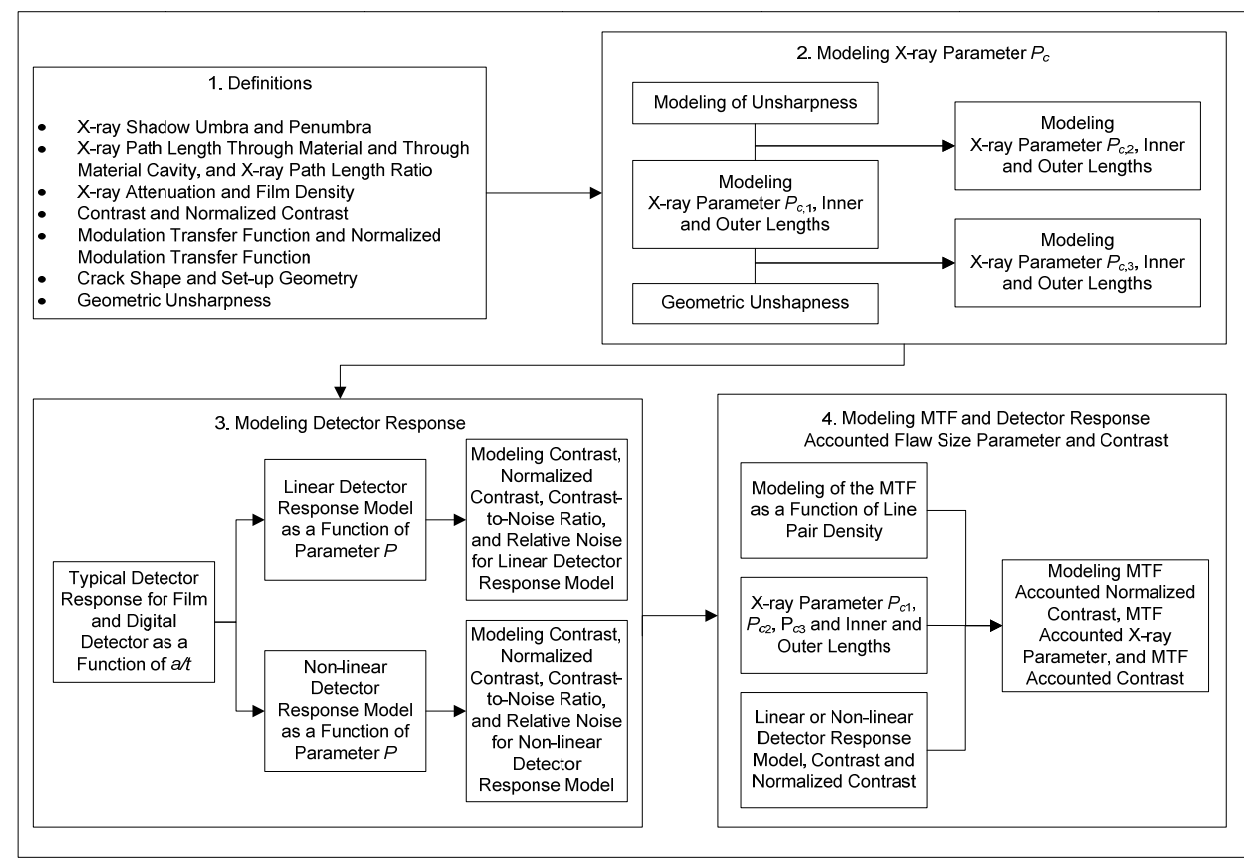

Fig. 1: Flow chart for development of the model

\section{MODELING CRACK SHAPE AND SETUP GEOMETRY}

Here, we take a single open-to-surface rectangular slot as the crack in a plate. The slot depth is denoted by $a$, and width or opening is denoted by $W$. The plate thickness is denoted by $t$. The slot is normal to the plate surface. The imaging detector can be a film, computed radiography (CR) screen or any of the variety of digital detector (DR) panels or cameras. See Fig. 2 for cross sectional profile of the slot. The X-ray beam is in the plane of figure. The slot length and plane of detector plate are normal to the plane of figure. We assume that an estimate of crack width is available. The material thickness under the slot is denoted by $b$.

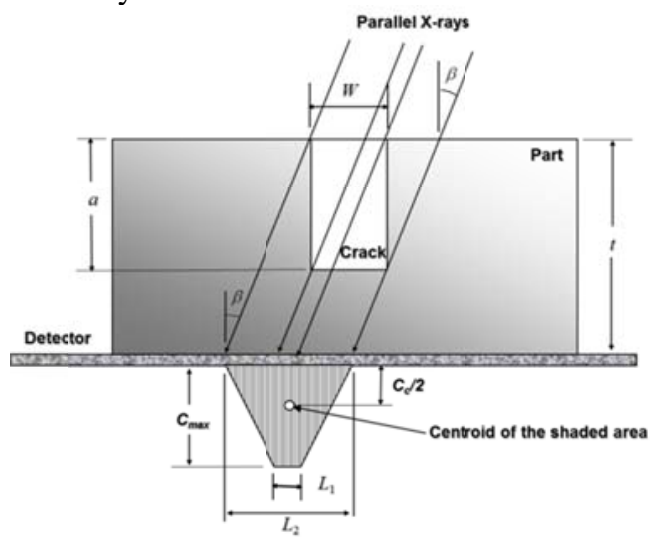

Fig. 2: Part, slot, detector geometry, selected X-rays and X-ray shadow profile

We also use a step wedge ${ }^{5}$ made from the plate material for calibration of the signal response. See Fig. 3 . Thickness of the thickest step of the wedge is same as the plate thickness. Thickness of each step is then denoted by $b$ and depth of each step from top of the highest step is denoted by $a$.

We also use line-pair resolution targets ${ }^{5}$ to characterize the modulation transfer function of the detector as applicable to the X-ray test set-up. See Fig. 3. The plate with a slot, the step wedge and the line-pair resolution target are subjected to the same X-ray inspection set-up. Slot depth $a$, material thickness under slot $b$ and plate thickness $t$ are related by, 


$$
b=t-a \text { or } a=t-b .
$$

Since the flaw detection is based on image contrast of the flaw indication, we intend to formulate the flaw size parameter that relates to the image contrast.

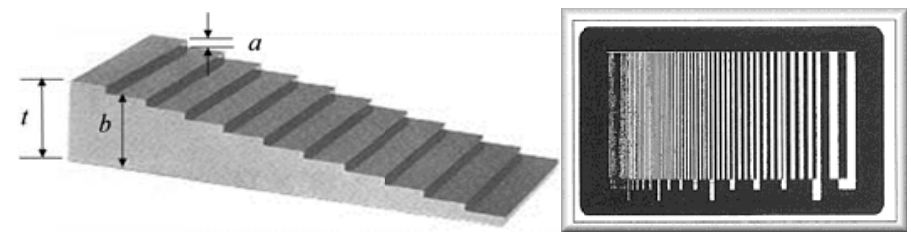

Fig. 3: An aluminum step wedge and a line pair resolution target made from a $0.05 \mathrm{~mm}$ thick lead shim

\section{X-RAY ATTENUATION AND FILM DENSITY}

Let us first consider a radiography setup where a plate of thickness $t$ is radiographed. We assume that the X-rays are normal to the top surface of the plate and the detector is in intimate contact with bottom of the plate. If intensity of Xrays entering the plate is $I_{0}$ and intensity of X-rays exiting the plate is $I_{t}$, then the two intensities are related by ${ }^{6,7,8}$,

$$
I=I_{0} e^{-\alpha t} \text { or } \ln I_{t}=\ln I_{0}-\alpha t \text {, }
$$

where,

$I_{0}=$ X-ray intensity or exposure incident on top side the of plate,

$I_{t}=\mathrm{X}$-ray intensity or exposure exiting from bottom side the of plate, and

$\alpha=$ X-ray attenuation coefficient.

The above equation is valid for monochromatic X-radiation and is assumed to be approximately valid for X-ray tubes used in the nondestructive evaluation. X-ray intensity is measured in rem per unit area per unit time. Exposure or absorbed energy dose is measured in rem ${ }^{6,7}$. However, in industrial radiography, the exposure is defined differently. It is defined as the tube current times the shot duration ${ }^{6}$. The source energy, source-to-part and part-to-detector distances shall be noted, as change in the source-to-detector distance would affect the exposure. We use the X-ray intensity and exposure (tube current times the shot time) interchangeably because the $\mathrm{X}$-ray intensity multiplied by area and time is proportional to the exposure in a given set-up ${ }^{6}$.

$\mathrm{X}$-ray film density is measured by noting fraction of light transmitted through the film. The film density is defined by the following expression ${ }^{6}$,

$$
d=\log _{10} \frac{I_{0}^{f}}{I_{1}^{f}},
$$

where,

$I_{0}^{f}=$ intensity of light incident on the film,

$I_{1}^{f}=$ intensity of light transmitted through the film,

$d=$ film density. Film density correlates better with logarithm of the X-ray exposure.

\section{IMAGE CONTRAST}

Result of an X-ray inspection is a digital image or film with an image of the slot. The slot is identified in the image due to image contrast. Contrast or net detector response is defined as ${ }^{6,7}$,

$$
S_{a}=J_{a}-J_{0},
$$

where,

$J_{a}=$ detector response registered at the slot image location and

$J_{0}=$ detector response registered in the surrounding region of plate thickness $t$ (or at $a=0$ ). 
In the above equation, we assume that the slot indication has a higher numerical value for image intensity or film density than in the surrounding region. See Fig. 4 for an example of the net detector response for a film obtained on a step wedge as a function of step depth-to-part thickness ratio $(a / t)$. The data-points have been joined by a smooth curve.

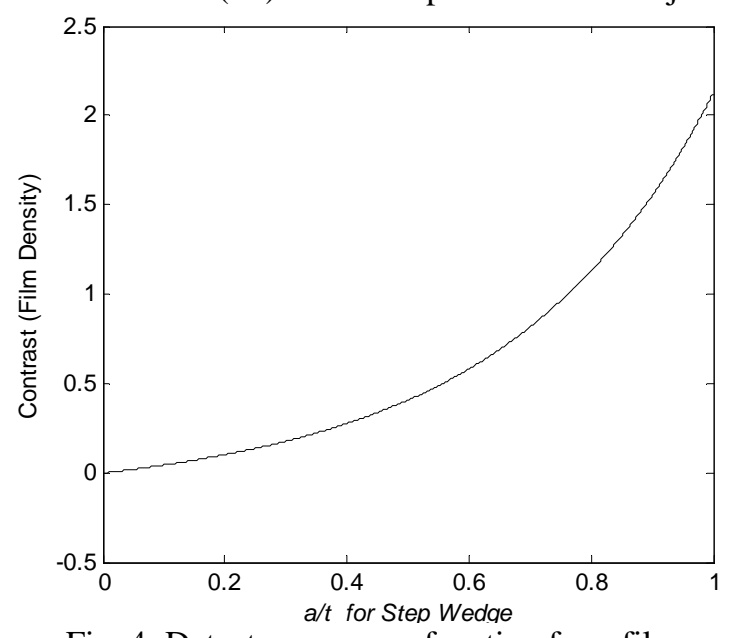

Fig. 4: Detector response function for a film

Here, we introduce normalized contrast, which is based on the net detector response. Net detector response due to a slot or step of depth $t$ in a plate of thickness $t$ is given by,

$$
S_{t}=J_{t}-J_{0},
$$

where,

$J_{t}=$ detector response at $a=t$ (i.e. plate is absent).

By definition, the net detector response for $a=0$ is zero. $S_{0}=J_{0}-J_{0}=0$. The normalized contrast or normalized detector response, as defined here, is given by,

$$
C=\frac{S_{a}}{S_{t}} .
$$

See Fig. 5 for an example of the normalized detector response or normalized contrast for a digital detector obtained on a step wedge as a function of step depth-to-part thickness ratio, e.g. $a / t$. It is assumed that an adequate exposure is used so that the signal-to-noise $(\mathrm{S} / \mathrm{N})$ ratio is high.

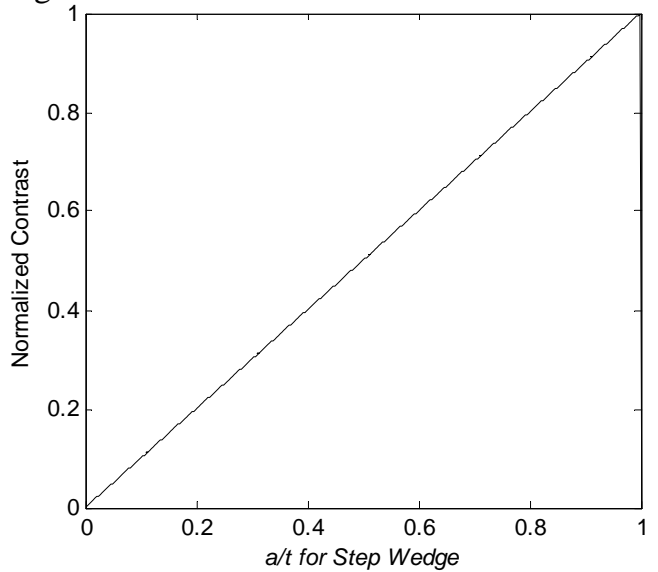

Fig. 5: Normalized detector response function for a digital detector

\section{LINEAR DETECTOR RESPONSE MODEL}


Let us consider a linear model of the detector response first. We assume that the detector contrast is approximately linear with natural logarithm of the exposure ${ }^{6,7}$. Using eq. (2) for logarithm of exposure, the detector response is modeled here as,

$$
J_{0}=q_{1}\left(\ln I_{0}-\alpha t-q_{3}\right)+q_{2}+\delta,
$$

where,

$J_{0}=$ detector response due to X-ray exposure on a plate of thickness $t$,

$q_{1}=$ detector sensitivity,

$q_{2}, q_{3}=$ detector coefficients, and

$\delta=$ detector noise.

Detector noise is assumed to be distributed normally with zero mean. Standard deviation of the detector noise is denoted by $\sigma_{\delta}$. We assume that the detector response is averaged in the background. If an average value is used for $J_{0}$, then the noise term $\delta$ is not used. The detector does not respond until it receives a minimum level of exposure. Also, the response is saturated above a certain level of exposure. Thus, the linear model is valid only between these extremities. The digital detectors have a higher dynamic range and respond to a relatively low exposure as well as high exposure with a linear relationship throughout the range. The X-ray film has a lower dynamic range compared to the dynamic range of an amorphous-silicon digital detector. Relationship between the film density and exposure is non-linear. Relationship between the digital detector response and exposure is approximately linear. For convenience we would omit the noise term in the following five equations. By substituting step thickness $b$ and $t$ separately in eq. (7), various detector response expressions are derived as,

$$
\begin{gathered}
J_{a}=q_{1}\left(\ln I_{0}-\alpha b-q_{3}\right)+q_{2}, \\
J_{a}=q_{1}(\alpha a)+J_{0}, \text { and } \\
J_{t}=q_{1}(\alpha t)+J_{0} .
\end{gathered}
$$

Here, we assume that the measurement is done on an indication of a step in a step wedge or on a wide slot in a plate. By rearranging eq. (9), the contrast or net detector response at a step is given by,

$$
S_{a}=J_{a}-J_{0}=q_{1} \alpha a .
$$

By rearranging eq. (10), the net detector response for the plate is given by,

$$
S_{t}=J_{t}-J_{0}=q_{1} \alpha t \text {. }
$$

By substituting eq. (11) and (12) in eq. (6) and neglecting noise, the ideal normalized contrast is given by,

$$
C=\frac{S_{a}}{S_{t}}=a / t .
$$

However, in practice the contrast measurement would be affected by noise. Following expression indicates effect of the detector noise $\delta(\mu, \sigma)$ in measuring contrast.

$$
C=\frac{q_{1} \alpha a+\delta}{q_{1} \alpha t} .
$$

Here, the noise term is neglected in the denominator of the above expression by assuming averaging of intensities over appropriate areas in measuring $S_{t}$. Contrast and normalized contrast are assumed to relate to flaw detection ability. The above equation is independent of $J_{0}$ but assumes that logarithm of the exposure provides a response that is within the linear response range. The above equation implies that higher step (or slot) depth-to-part-thickness ratio provides higher normalized contrast.

Contrast-to-noise ratio ${ }^{9}$ is given by,

$$
C N R=\frac{S_{a}}{\sigma_{\delta}}=\frac{q_{1} \alpha a}{\sigma_{\delta}} .
$$

The above equation implies that higher material attenuation coefficient, higher detector sensitivity and slot depth provide better signal-to-noise ratio. A high contrast-to-noise ratio, e.g. greater than 3, is desired for reliable detection of flaws. Relative noise, as defined here, is given by, 


$$
R_{\text {noise }}=\frac{\sigma_{\delta}}{J_{0}} .
$$

Detector noise is used in noise analysis which is used to supplement the POD analysis by providing an estimate of false call rate. Detector exposure factors and contrast-to-noise ratio are better addressed in the contrast than in the normalized contrast. Normalized contrast is related to flaw detection and it is less dependent upon the exposure, X-ray attenuation coefficient and detector characteristics. Later, ratio a/t is extended for narrow width slots by defining the X-ray parameter $P$ that is related to the flaw contrast.

\section{NON-LINEAR DETECTOR RESPONSE MODEL}

An example of a non-linear model is given in Fig. 4. By neglecting detector noise, and assuming a typical nonlinear detector response as shown in Fig. 4, a general non-linear model can be given as,

$$
\begin{gathered}
J_{a}=f_{n}(a / t)+J_{0}, \\
J_{t}=f_{n}(1)+J_{0}, \\
S_{a}=J_{a}-J_{0}=f_{n}(a / t), \\
f_{n}(0)=0,
\end{gathered}
$$

where,

$J_{a}=$ detector response due to X-ray exposure to plate of thickness $b$,

$f_{n}(a / t)=$ a function or dependent variable of $a / t$, and

$J_{0}=$ detector response when $a=0$ in a part thickness of $t$.

Ideal normalized contrast is given by,

$$
C=\frac{f_{n}(a / t)}{f_{n}(1)} .
$$

However, in practice the contrast measurement would have the effect of noise. Following expression indicates how contrast is affected by the detector noise.

$$
C=\frac{f_{n}(a / t)+\delta}{f_{n}(1)} .
$$

\section{MODULATION TRANSFER FUNCTION}

Modulation transfer function (MTF) as a function of line-pairs per unit distance or spatial frequency of an image target is commonly used to characterize resolution of an imaging array or film type detector. A line-pair in an imaging target is defined by a dark line and a light line of equal width $L$ placed next to it ${ }^{6,7}$. The line-pair width in the target is given by,

$$
L_{l p}=2 L \text {. }
$$

Line-pair frequency measured in line-pair per mm is denoted by $w_{l p}$. The line-pair frequency and the line-pair width are related by,

$$
w_{l p}=\frac{1}{L_{l p}} .
$$

We assume that the width $L_{l p}$ is given in millimeter. A 2D digital detector array panel has square pixels with fixed pitch between pixels. The pitch or the detector pixel width can be taken as the detector width $L_{d}$.

The line-pair resolution is also applicable for X-ray inspection. In the X-ray inspection, a line-pair target is made from a thin lead shim. See Fig. 3. Many pairs of shim-gap with same width are grouped so that the image width of any line-pair group is longer than the detector pixel size.

Modulation transfer function can be calculated by imaging the line pair resolution target and using the following equation. 


$$
M=\frac{J_{t}^{\prime}-J_{0}^{\prime}}{J_{t}^{\prime}+J_{0}^{\prime}},
$$

where,

$J_{0}^{\prime}=$ minimum film density or image intensity of the shim indication, and

$J_{t}^{\prime}=$ maximum film density or image intensity of the gap indication.

In the above equation, we assume that the gap indication has higher numerical value of image intensity than the shim indication. MTF is plotted and modeled as a dependent variable of $w_{l p}$ or $L_{l p}$ of the imaging target at the detector. When an indication is very wide i.e. more than ten times of the detector element size, the registered intensity is not altered or modulated due to slight change in the indication width. The maximum MTF is given by,

Normalized MTF is given by ${ }^{10}$,

$$
M_{\max }=\frac{J_{t}-J_{0}}{J_{t}+J_{0}} .
$$

$$
M_{n}=\frac{M}{M_{\max }} .
$$

Sum of the shim and gap indication modulated intensities is same as that of unmodulated intensities. Therefore,

$$
J_{t}^{\prime}+J_{0}^{\prime}=J_{t}+J_{0} .
$$

Therefore, by combining eq. (26) and (27) the modulated intensities relate to the unmodulated intensities as,

$$
J_{t}^{\prime}-J_{0}^{\prime}=M_{n}\left(J_{t}-J_{0}\right) \text {. }
$$

Sum of the modulated intensities is same as that of unmodulated intensities. Therefore,

$$
J_{a}^{\prime}+J_{0}^{\prime}=J_{a}+J_{0} .
$$

By combining eq. (27) and (29) the modulated intensities relate to the unmodulated intensities as,

$$
J_{a}^{\prime}-J_{0}^{\prime}=M_{n}\left(J_{a}-J_{0}\right) .
$$

The modulated signal response at the slot for a line-pair resolution target is given by,

$$
J_{a}^{\prime}=\frac{J_{a}+J_{0}+M_{n}\left(J_{a}-J_{0}\right)}{2} .
$$

The above equation is true for imaging a line-pair target. MTF may also be a function of angle of the incident radiation. If MTF is used in the analysis, then detector unsharpness $U_{d}$ should not be used. The normalized MTF is modeled as a function $g_{\mathrm{n}}$ of line-pair frequency or width.

$$
\begin{aligned}
& M_{n}=g_{n}\left(w_{l p}\right) \text { or } \\
& M_{n}=g_{n}\left(1 / L_{l p}\right) .
\end{aligned}
$$

A curve may be modeled in the MTF plot. For example, the modulation transfer function may be modeled as,

$$
g_{n}\left(w_{l p}\right)=a_{1} e^{a_{2} w_{l p}}+a_{3} e^{a_{4} w_{l p}},
$$

where, $a_{1}, a_{2}, a_{3}$ and $a_{4}$ are the coefficients.

Examples of assumed MTF for two different detectors are given in Fig. 6 and 7. Here, we choose a line-pair resolution corresponding to an MTF value of 0.4 as the line-pair resolution $\left(L_{d}\right)$ of the detector. The detector resolution is assumed to relate to the pixel size of a digital radiographic detector or average grain size of a radiographic film. Another MTF value (e.g. 0.2) can be chosen. The chosen MTF value allows us to quantify the resolution of a detector in terms of linepair frequency.

MTF value for a film is 0.4 for a $20 \mathrm{lp} / \mathrm{mm}$ target as shown in Fig. 6. 

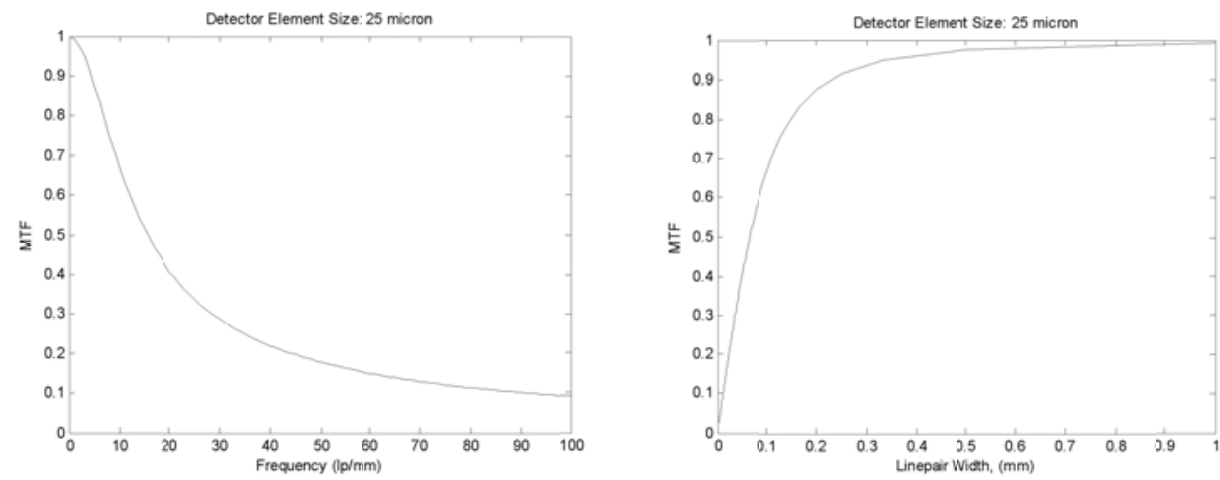

Fig. 6: MTF used for the film in the simulation

MTF value for a digital detector is 0.4 for a $4.2 \mathrm{lp} / \mathrm{mm}$ target as shown in Fig. 7.
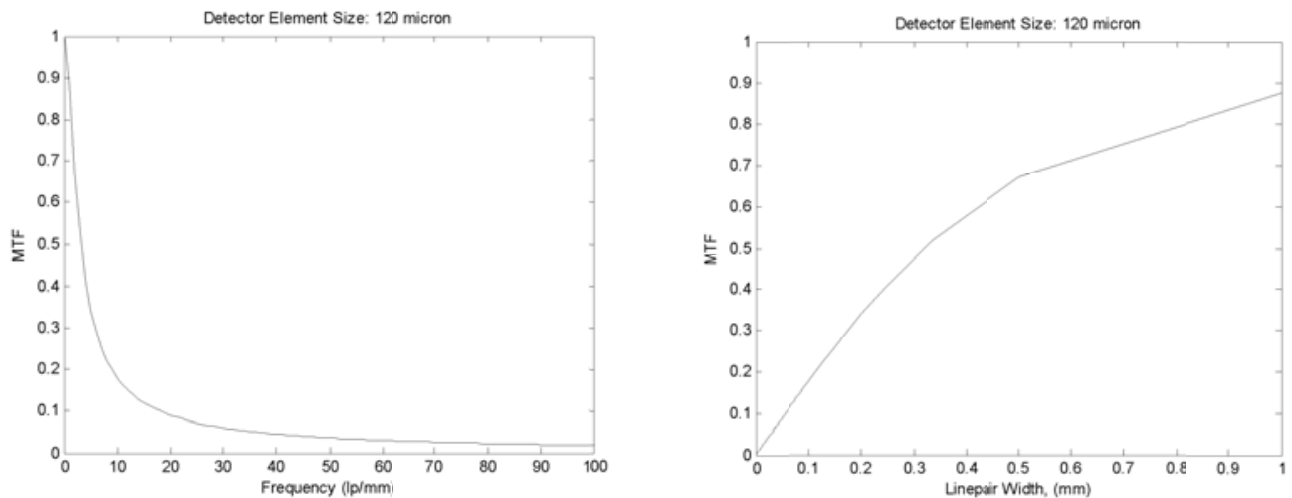

Fig. 7: MTF used for the digital detector in the simulation

By substituting the flaw size parameter $P_{\mathrm{c}}$, defined later, in place of $a / t$ in eq. (21) and multiplying by the normalized MTF we get MTF accounted normalized contrast parameter.

$$
C_{f(P), M}=M_{n} \frac{f_{n}\left(P_{c}\right)}{f_{n}(1)}=g_{n}\left(L_{l p}\right) \frac{f_{n}\left(P_{c}\right)}{f_{n}(1)} .
$$

If the detector response is not known, then the normalized contrast parameter based on the X-ray parameter and MTF is given as,

$$
C_{c, M}=M_{n} P_{c}=g_{n}\left(1 / L_{l p}\right) P_{c} .
$$

Here, we rewrite the above equation as,

$$
P_{c, M}=M_{n} P_{c}=g_{n}\left(1 / L_{l p}\right) P_{c} .
$$

The above quantity can also be considered to be modulation accounted X-ray parameter. The above two equations relate to the normalized contrast of a single slot when modulation effect of the detector is accounted. Modulation accounted contrast is given by,

$$
S_{f\left(P_{c}\right), M}=M_{n} f_{n}\left(P_{c}\right)=g_{n}\left(1 / L_{l p}\right) f_{n}\left(P_{c}\right) .
$$

Similarly, it is assumed that the shadow contrast $f_{n}(P)$ is uniform within the shadow width.

\section{MODEL BASED ON THE X-RAY PARAMETER}

Visual perception of the slot indication is primarily influenced by the image contrast. The perception is affected by background intensity to a lesser extent. Visual perception is not good if images are too dark or too bright. Therefore, in film evaluation, the viewing light intensity is controlled by a backlight illuminating the film. In digital images, the image processing software allows changing contrast (and brightness) by rescaling within a chosen viewing intensity range. Base 
intensity should also have good signal-to-noise ratio. Typically, contrast-to-noise ratio of greater than 3 is needed to detect flaws reliably.

Ratio $a / t$ is a measure of the ideal normalized contrast. Therefore, ratio $a / t$ can be considered to be a fraction of the X-ray pathlength passing through void of the slot. Ratio a/t can be described as the "void pathlength ratio". During inspection, an X-ray penetrameter that is made from the plate material is placed on top of the plate. A 2-2T hole of the strip penetrameter ${ }^{10}$ provides a void pathlength ratio of 0.0196 or approximately $2 \%$.

If the 2-2T hole or the penetrameter edge can be identified in an X-ray image, it does not imply that a crack with $2 \%$ depth can be imaged or detected in the radiographic image. This is due to combined effect of the crack related factors and technique related factors. Some of the technique related factors include incident angle of X-rays, geometric unsharpness and resolution of the recording medium. Some of the crack related factors include crack depth, length, opening, straightness of crack faces, and roughness of crack faces.

The model uses a parameter based on the spatial variation of the X-ray pathlength ratio through the crack void as mapped on the recording medium. Contrast is also affected by geometric unsharpness and other factors. Therefore, the X-ray parameter is based on assessing average effect of the X-ray pathlength ratio that also includes the effect of geometric unsharpness in the image of slot. Spatial variation of the pathlength ratio through the slot, in combination with unsharpness effects, is called the X-ray shadow profile here. We do not intend to provide an algorithm to compute shadow intensity for every point in the shadow profile. We assess an equivalent intensity for the slot shadow in the X-ray parameter. Maximum possible value of the X-ray parameter is $a / t$.

Geometric unsharpness causes edges of the shadow to be blurred. Zone of blurred edge of the shadow is called penumbra. Middle or inner portion of the shadow is called umbra. Width measured between the outer edges of penumbra provides the outer shadow width. Equivalent shadow width $L_{e}$ is defined such that the product of X-ray parameter and equivalent length is $\mathrm{Wa} / \mathrm{t}$, which is the area under the shadow profile. We use the $\mathrm{X}$-ray parameter and associated equivalent width in evaluation of the normalized contrast and contrast.

In reality, X-rays may have an oblique angle to the crack face. The crack opening may be very narrow. These factors would decrease the X-ray parameter below its maximum possible value of $a / t$. The X-ray parameter is used in the MTF accounted normalized contrast and contrast as follows.

$$
\begin{gathered}
C_{f\left(P_{c}\right), M}=g_{n}\left(1 / 2 L_{e}\right) \frac{f_{n}\left(P_{c}\right)}{f_{n}(1)}, \text { and } \\
S_{f\left(P_{c}\right), M}=g_{n}\left(1 / 2 L_{e}\right) f_{n}\left(P_{c}\right) .
\end{gathered}
$$

Consider a special case of an ideal detector where the detector response is linear,

$$
f_{n}\left(P_{c}\right)=k P_{c} \text { and } f_{n}(1)=k \text {. }
$$

Here, $k$ is the detector sensitivity constant in a given inspection set-up. Note that $k$ also depends upon the X-ray material attenuation coefficient. Substituting eq. (42) in eq. (40), we get,

$$
C_{f\left(P_{c}\right), M}=g_{n}\left(1 / 2 L_{e}\right) P_{c} .
$$

We would rewrite the above equation as,

$$
P_{f\left(P_{c}\right), M}=g_{n}\left(1 / 2 L_{e}\right) P_{c} .
$$

The above quantity can also be considered to be the modulation accounted X-ray parameter. The above equation relates to the contrast of the line-pair resolution target but also relates to normalized contrast due to a single slot. Eq. (41) is rewritten as,

$$
S_{f\left(P_{c}\right), M}=g_{n}\left(1 / 2 L_{e}\right) k P_{c} .
$$

Contrast for a single slot is affected by the detector pixel size. The above two equations indicate that, when the net detector response is linear with $a / t$, the contrast and the normalized contrast are proportional to a product of the X-ray parameter $P$ and MTF value for the shadow width. A high MTF value is obtained if the detector has high resolution in comparison to the shadow width $L_{e}$. A high value of detector sensitivity $k$, near the highest possible value for the technique, is desired to maximize the contrast. Normally, radiographic film sensitivity is high for a film density of 2.5 to 
4. The radiographic technique (energy, filters, duration, density) can be controlled such that a desired value of the detector sensitivity $k$ can be obtained.

The above equations indicate that the X-ray parameter relates to the contrast which is assumed to relate to the flaw detection. Therefore, the X-ray parameter relates to the flaw detectability or POD, provided the detector sensitivity and detector resolution are controlled or standardized. The technique sensitivity can be assessed by radiographing and evaluating a resolution target and step wedge. We would further develop the X-ray parameter so that it relates to some more set-up geometry related parameters, including source-to-part distance, part-to-detector distance, X-ray angle, source size, slot width, slot depth, geometric and other unsharpness.

\section{MODEL LEVEL 1: PARALLEL X-RAYS}

In developing a model for the X-ray parameter, we start with the simplest configuration. Here, we assume that the source provides parallel rays in level 1 model. A crack is modeled as a slot described earlier. See Fig. 2. Two modes, e.g. mode 1 and mode 2, are defined here depending on interaction of a parallel X-ray beam with cross sectional geometry of the slot. Mode 1 is defined by the following condition,

$$
W \geq a \tan \beta \text {, }
$$

where,

$\beta=$ angle of $\mathrm{X}$-rays with respect to normal to the part surface.

When this condition is satisfied, some X-rays entering the slot opening on top side pass through flat bottom of the slot. In mode $1, P_{\max , 1}$ is given by,

$$
P_{\text {max }, 1}=a / t,
$$

where,

$P_{\max , 1}=$ peak value of the $\mathrm{X}$-ray shadow profile. See Fig. 2 for a schematic of the $\mathrm{X}$-ray shadow profile.

Subscript "1" is used to indicate the model level. A slot of width $W$ casts an image with length given by $L_{2,1}$,

$$
L_{2,1}=W+a \tan \beta \text {. }
$$

$L_{2,1}$ is also called outer width of the shadow. Slot indication contrast is high in the center zone or umbra and it tapers in the side zone or penumbra. Width of the center or inner portion with higher contrast is given by $L_{1,1}$.

$$
L_{1,1}=W-a \tan \beta \text {. }
$$

$L_{1,1}$ is also called inner width of the shadow. Mode 2 is defined by the following condition,

$$
W<a \tan \beta \text {. }
$$

When this condition is satisfied, no X-rays entering from top of the slot opening pass through flat bottom of the slot opening. The maximum $\mathrm{X}$-ray shadow intensity is given by,

$$
P_{\max , 1}=\frac{W}{t \tan \beta} .
$$

In mode 2, outer width of the shadow is given by,

$$
L_{2,1}=W+a \tan \beta \text {. }
$$

In mode 2, inner width or width of the center portion of shadow is given by,

$$
L_{1,1}=a \tan \beta-W \text {. }
$$

There is a smooth transition between mode 1 and mode 2 as the X-ray angle increases. The transition point is defined by the following condition,

$$
W=a \tan \beta .
$$

Here, we generically define the two shadow widths as,

$L_{1, n}=$ umbra or inner shadow width. Subscript " $n$ " indicates the model level number and

$L_{2, n}=$ total or outer shadow width. The outer shadow width contains both umbra and penumbra shadow zones.

For both modes, the inner shadow width is given by,

$$
L_{1,1}=|a \tan \beta-W| .
$$

Human detection of a fine crack is assumed to be dependent not only on the contrast associated with peak of the shadow but also on indication widths $L_{1}$ and $L_{2}$. This assumption will not hold if $L_{1}$ and $L_{2}$ are about same or value of X-ray 
parameter is too high (e.g. possibly much greater than $2 \%$ ). But barring these extremities, the area under the shadow profile can be used to assess equivalent indication intensity. The $\mathrm{X}$-ray parameter is given by,

$$
P_{c}=P_{\max } \frac{2}{3}\left(\frac{2 L_{1}+L_{2}}{L_{1}+L_{2}}\right) .
$$

Subscripts indicating the model number have been dropped to indicate the general formula. Subscript " $c$ " is used to indicate that the X-ray parameter is based on the centroidal weighing or average i.e. centroidal distance (height) of shadow profile in order to assign a single value related to contrast perception. See Fig. 2. $P_{c}$ is defined as two times the height of centroid of the shadow profile in Fig. 2. The X-ray parameter is not used to calculate an estimated image contrast but is used to calculate an "equivalent" contrast that may be related to the actual contrast. Here, we can also use an approach to divide the area under the contrast curve by the average width of the indication to obtain a quantity related to contrast. While such approach may also work, the centroid is believed to provide a better weighing of the area under the contrast curve.

$P_{\mathrm{c}}$ is equal to twice the centroidal height of the indication. Thus, if $L_{1}$ and $L_{2}$ are equal, then $P_{\mathrm{c}}=P_{\max }$ and if the shadow profile has a triangular shape $\left(L_{1}=0\right)$, then $P_{\mathrm{c}}=(2 / 3) P_{\max }$. By substituting eq. (47) in eq. (56), for mode 1 we get,

$$
P_{c, 1}=\frac{2}{3}(a / t)\left(\frac{2 L_{1,1}+L_{2,1}}{L_{1,1}+L_{2,1}}\right) .
$$

By substituting eq. (51) in eq. (56), for mode 2 we get,

$$
P_{c, 1}=\frac{2}{3}\left(\frac{W}{t \tan \beta}\right) \frac{2 L_{1,1}+L_{2,1}}{L_{1,1}+L_{2,1}} .
$$

\section{MODEL LEVEL 2: FINITE SIZE SOURCE AND UNSHARPNESS}

Here, we begin work on a slightly more sophisticated level 2 model. In this model, we account for the unsharpness in a simple way. We define unsharpness as follows.

$$
U_{n}=U_{g}+U_{o}
$$

Here, subscript " $g$ " is used to indicate the geometric unsharpness. Unsharpness due to other effects, $U_{\mathrm{o}}$ is given by,

$$
U_{o}=U_{f}+U_{s}+U_{m} \text {. }
$$

Subscript " $f$ " is used to denote film effects, subscript " $s$ " is used to denote scatter effects, and subscript " $m$ " is used denote cumulative of other miscellaneous effects. Although, unsharpness components are identified, in most cases only geometric unsharpness is known. Other factors contributing to the unsharpness can be considered to be part of the conditions that would be controlled. Under these assumptions for mode 1 and 2, the inner shadow width is given by,

$$
L_{1,2}=|a \tan \beta-W|\left(\frac{d_{1}+d_{2}}{d_{1}}\right)-U_{n} \mid,
$$

where,

$d_{1}=$ source to part distance, and

$d_{2}=$ part to detector distance.

The above equation is derived by applying geometric enlargement to the inner shadow width of model 1 and then subtracting the net unsharpness. The outer shadow width is given by,

$$
L_{2,2}=(a \tan \beta+W)\left(\frac{d_{1}+d_{2}}{d_{1}}\right)+U_{n} .
$$

The above equation is derived by applying geometric enlargement to the outer shadow width of model 1 and then adding the net unsharpness. The approximate equivalent indication length for model 2 is given by,

$$
L_{e, 2}=\frac{a W}{t} \frac{1}{P_{c, 2}} .
$$

$\mathrm{X}$-ray parameter for mode 1 in model 2 is given by, 


$$
P_{c, 2}=\frac{2}{3}\left(\frac{a}{t}\right)\left(\frac{2 L_{1,2}+L_{2,2}}{L_{1,2}+L_{2,2}}\right)\left(\frac{L_{1,1}+L_{2,1}}{L_{1,2}+L_{2,2}}\right) .
$$

The above equation is similar to corresponding equation for model 1 , except the increase in length of the indication due to geometric enlargement and unsharpness is accounted by proportionately reducing the X-ray parameter. Similarly, Xray parameter for mode 2 in model 2 is given by,

$$
P_{c, 2}=\frac{2}{3}\left(\frac{W}{t \tan \beta}\right)\left(\frac{2 L_{1,2}+L_{2,2}}{L_{1,2}+L_{2,2}}\right)\left(\frac{L_{1,1}+L_{2,1}}{L_{1,2}+L_{2,2}}\right) .
$$

\section{MODEL LEVEL 3: FINITE SIZE SOURCE AND UNSHARPNESS}

Here, we begin work on model 3 which will be slightly more sophisticated than model 2 but provides the same quantities with slightly different equations. Similar to model 2, model 3 is derived from model 1 . In model 3 , the unsharpness is handled differently. Unsharpness is handled as mainly contributed by the geometric unsharpness. Geometric unsharpness is given by,

$$
U_{g}=\frac{S d_{2}}{d_{1}},
$$

where,

$S$ = width of the X-ray source.

Here, we make an assumption that the slot can be replaced by an equivalent shadow in the shape of a rectangle with height given by,

$$
P_{x, 3}=P_{c, 1}=\frac{2}{3} P_{\max , 1} \frac{\left(2 L_{1,1}+L_{2,1}\right)}{\left(L_{1,1}+L_{2,1}\right)},
$$

and the equivalent width is given by $L_{\mathrm{x}}$.

$$
L_{x, 3}=\frac{3}{4} \frac{\left(L_{1,1}+L_{2,1}\right)^{2}}{\left(2 L_{1,1}+L_{2,1}\right)} .
$$

$L_{x}$ is derived by keeping area under the shadow profile same as in model 1 . The above equation provides a length between $L_{1,1}$ and $L_{2,1}$ The length is approximately given by $L_{1,1}+(2 / 3)\left(L_{2,1}-L_{1,1}\right)$.

The simulated slot shadow with width, $L_{x, 3}$ is then projected onto the imaging plane using X-rays from the source.

In this situation, the outer and inner widths of the shadow are,

$$
\begin{gathered}
L_{2,3}=\frac{L_{x, 3}\left(d_{1}+d_{2}\right)}{d_{1}}+U_{n}, \text { and } \\
L_{1,3}=\left|\frac{L_{x, 3}\left(d_{1}+d_{2}\right)}{d_{1}}-U_{n}\right| .
\end{gathered}
$$

We assume that area under the shadow profile is same between a parallel beam source and a source with known size. Therefore,

$$
\begin{gathered}
P_{\max , 3}\left(L_{1,3}+L_{2,3}\right)=P_{\max , 1}\left(L_{1,1}+L_{2,1}\right), \text { or } \\
P_{\max , 3}=P_{\max , 1} \frac{L_{1,1}+L_{2,1}}{L_{1,3}+L_{2,3}} .
\end{gathered}
$$

Using eq. (56), X-ray parameter is given by,

$$
P_{c, 3}=\frac{2}{3} P_{\max , 3} \frac{2 L_{1,3}+L_{2,3}}{L_{1,3}+L_{2,3}} .
$$

Equivalent shadow width is given by,

$$
L_{e, 3}=\frac{a W}{t} \frac{1}{P_{c, 3}} \text { or }
$$




$$
L_{e, 3}=\frac{3}{4} \frac{\left(L_{1,3}+L_{2,3}\right)^{2}}{\left(2 L_{1,3}+L_{2,3}\right)} .
$$

The equations for model 3 are slightly different for equivalent width than those of model 2. One of the two models (e.g. model 2 or 3) is likely to be slightly better depending upon magnitudes of geometric unsharpness, other unsharpness and geometric enlargement.

\section{DETECTOR ORIENTATION AND OTHER FACTORS}

Here, we consider an angle $\alpha$ between the detector plane and plane of the plate. Angle between the detector and the plate increases width of the shadow on the detector. The corresponding shadow lengths are,

$$
\begin{gathered}
L_{1,4}=L_{1,3} \sec \alpha, \text { and } \\
L_{2,4}=L_{2,3} \sec \alpha .
\end{gathered}
$$

The larger shadow width is advantages in detecting smaller flaws but the relative noise increases. Moreover, the detector may be less sensitive to obliquely incident $\mathrm{X}$-rays. The $\mathrm{X}$-ray parameter is unaffected, i.e.,

$$
P_{c, 4}=P_{c, 3} \text {. }
$$

The average shadow width is given by,

$$
L_{e, 4}=L_{e, 3} \sec \alpha .
$$

Equivalent width is useful to model detector resolution effects. We also consider the unsharpness $U_{d}$ at the detector screen, especially if X-ray radiation is not normal to the detector. Detector unsharpness may be a function of angle of the incident radiation. Equivalent width of the shadow at the detector and the X-ray parameter are given by,

$$
\begin{gathered}
L_{e, 5}=L_{e, 4}+U_{d} \text {, and } \\
P_{c, 5}=P_{c, 4} \frac{L_{e, 4}}{L_{e, 5}} .
\end{gathered}
$$

\section{USE OF X-RAY PARAMETERS AND SIMULATED CONTRAST}

The X-ray parameters can be used in the POD studies. The crack widths or openings should be measured using a microscope. Destructive microscopy on a few cracks will be useful in estimating crack widths and crack length-to-depth ratio. The X-ray technique sensitivity is assumed to be controlled and maintained at its nominal value using measurements on a step wedge and a resolution standard. Experimental correlation of the signal response with the X-ray parameter $\left(P_{c}\right.$ or $P_{c, M}$ ) or success/failure detection result for a range of $\mathrm{X}$-ray parameters should be obtained. Contrast-tonoise ratio should be noted. An appropriate image processing or filtering routine should be applied to the entire dataset during a POD study. Routines that maintain correlation to the image contrast are useful in "â" versus "a" POD analysis else the processed images can be used in "hit-miss" POD analysis. A POD curve can be established with the X-ray parameter as the independent variable. The results may establish an a90/95 in terms the X-ray parameter for a given Xray technique and material. Alternately, the simulated contrast $(S)$ or the normalized contrast $(C)$ can be calculated and correlated with the measured contrast $(S$ and/or $C$ ) or with the flaw detection success/failure result. A POD curve can be established with the simulated contrast $(S$ or $C$ ) as the independent variable.

\section{COMPUTER APPLICATION}

A computer application or calculator called "X-Ray Parameter" is developed based on the 3 models. An example of MTF is simulated within the application. It allows choice of detector line-pair resolution. The detector calibration sensitivity $k$ can be input along with the source-part-detector geometry. The calculator input and output are illustrated for two cases in Table 1 . The data and results for a 70\% deep slot with X-ray angle of 5 degree are shown in Table 1 as Case 1. Table 1 also shows results of a $2 \%$ deep slot that is $2 \%$ wide similar to a $2-2 \mathrm{~T}$ penetrameter with X-ray angle of 5 degree as Case 2. $k$ value is arbitrarily taken to be 40,000 . The X-ray parameter and contrast values are about same between both cases. The comparison indicates that the $70 \%$ slot provides a contrast value equivalent to that of a $2-2 \mathrm{~T}$ penetrameter hole which a requirement in standard film X-ray. The X-ray parameter values are about $1.2 \%$ in the two cases. In the above example, the model 2 and 3 outputs are comparable. 3D graphical display of results for additional input data was omitted due to page length restrictions. 
Table 1: X-ray parameter calculation with examples of a 70\% deep slot and 2-2T slot

\begin{tabular}{|c|c|c|c|c|c|c|c|c|c|}
\hline Input Quantities & $\begin{array}{l}\text { Input } \\
\text { Case } 1\end{array}$ & $\begin{array}{c}\text { Input } \\
\text { Case } 2\end{array}$ & Output Quantities & $\begin{array}{c}\text { Case } 1 \\
\text { Model } 1\end{array}$ & $\begin{array}{c}\text { Case } 1 \\
\text { Model } 2\end{array}$ & $\begin{array}{c}\text { Case } 1 \\
\text { Model } 3\end{array}$ & $\begin{array}{c}\text { Case } 2 \\
\text { Model } 1\end{array}$ & $\begin{array}{c}\text { Case } 2 \\
\text { Model } 2\end{array}$ & $\begin{array}{c}\text { Case } 2 \\
\text { Model } 3\end{array}$ \\
\hline Crack Width $(W, \mathrm{~mm})$ & 0.005 & 0.08 & $\begin{array}{c}\text { Umbra Shadow Width } \\
\left(L_{1}, \mathrm{~mm}\right)\end{array}$ & 0.23997 & 0.23997 & 0.24603 & 0.07300 & 0.07169 & 0.08113 \\
\hline Crack Depth $(a, \mathrm{~mm})$ & 2.8 & 0.08 & $\begin{array}{l}\text { Net Shadow Width }\left(L_{2} \text {, }\right. \\
\text { mm) }\end{array}$ & 0.24997 & 0.25257 & 0.24923 & 0.08700 & 0.08895 & 0.08433 \\
\hline Part Thickness $(t, \mathrm{~mm})$ & 4 & 4 & $\begin{array}{l}\text { Equivalent Shadow } \\
\text { Width }\left(L_{\mathrm{e}}, \mathrm{mm}\right)\end{array}$ & 0.24665 & 0.24595 & 0.24817 & 0.08240 & 0.08032 & 0.08327 \\
\hline Source Width $(s, \mathrm{~mm})$ & 0.04 & 0.04 & $\mathrm{X}$-ray Parameter $\left(P_{\mathrm{c}}\right)$ & 0.01419 & 0.01410 & 0.01410 & 0.01942 & 0.01921 & 0.01921 \\
\hline $\begin{array}{c}\text { Source-to-Part Distance, } \\
\left(d_{1}, \mathrm{~mm}\right)\end{array}$ & 1,000 & 1,000 & $\operatorname{MTF}(M)$ & 0.85895 & 0.85862 & 0.85966 & 0.61321 & 0.60758 & 0.61547 \\
\hline $\begin{array}{l}\text { Part-to-Detector Distance } \\
\left(d_{2}\right)\end{array}$ & 4 & 4 & $\mathrm{X}$-ray Parameter $\left(P_{\mathrm{c}, \mathrm{M}}\right)$ & 0.01219 & 0.01211 & 0.01212 & 0.01191 & 0.01167 & 0.01183 \\
\hline Unsharpness $\left(u_{0}\right)$ & 0.00 & 0.00 & $\begin{array}{c}\text { Contrast ( } S_{f(P), M} \text {, gray } \\
\text { value) }\end{array}$ & 488 & 484 & 485 & 476 & 467 & 473 \\
\hline
\end{tabular}

\begin{tabular}{|c|c|c|}
\hline X-ray Angle $\left(\beta,^{\circ}\right)$ & 5 & 5 \\
\hline $\begin{array}{c}\text { Line Pair Frequency ( } w_{\mathrm{lp}}, \\
/ \mathrm{mm}) \text { @ MTF }=0.4\end{array}$ & 12 & 12 \\
\hline $\begin{array}{c}\text { Detector Sensitivity ( } k \text {, gray } \\
\text { value) }\end{array}$ & 40,000 & 40,000 \\
\hline
\end{tabular}

\section{CONCLUSIONS}

This approach assumes that the X-ray parameter and technique requirements including the part material requirements, the detector response calibration characteristics together provide a complete description the X-ray flaw detection application and provide sufficient information for assessment of the NDE capability. Although, the X-ray scatter is not modeled, it is assumed to be low, uniform and controlled by the technique, material, and detector requirements. The paper provides derivations of expressions for the X-ray parameters, normalized contrast, contrast, and shadow widths in an X-ray set-up for inspection of plate-like parts. It provides derivations that establish correlation between the X-ray parameters and contrast; and the X-ray parameter and normalized contrast. X-ray parameters defined here provide more advanced parameters for calculating $a 90 / 95$ than the currently used parameter $a / t$. A process of obtaining the detector response curve as a function of ratio a/t using a step wedge is described. Detector response curve is used in computation of the simulated contrast and simulated normalized contrast. The simulated contrast and simulated normalized contrast can be used for establishing correlation with actual contrast and flaw detectability. A computer calculator application, mentioned here, provides a simple way to evaluate whether an X-ray technique is capable of detecting given size flaws reliably by providing values of the X-ray parameters, contrast and normalized contrast that could be compared with corresponding a90/95 values that have been established for similar applications.

\section{ACKNOWLEDGEMENT}

Dr. Daniel Schneberk, Lawrence Livermore National Laboratory; Dr. Donald Roth, David Stanley and James Walker of National Aeronautics and Space Administration (NASA) provided valuable technical comments to this paper.

\section{REFERENCES}

[1] XRSIM, Commercial Software, NDE Technologies, Inc., HTTP://www.xrsim.com.

[2] CIVA Radiographic Module, Commercial software, http://www.extende.com/civa-2/civa-rt.

[3] Nondestructive Evaluation System Reliability Assessment, Department of Defense Handbook, MIL-HDBK-1823A, 2009.

[4] Improved Radiographic Inspection Capability for Detecting flaws, Report \# SD 76-SH-0196, F. E. Sugg, Rockwell International, 1976.

[5] Standard Practice for Evaluating the Imaging Performance of Security X-ray Systems, ASTM F 792-08.

[6] Industrial Radiography, AGFA NDT, AGFA-Gevaert, Inc., NDT \& Scientific Systems, 100 Challenger Road, Ridgefield Park, N.J. 07660, August 1987.

[7] Industrial Radiography: Image Forming Techniques, GE Inspection Technologies, GEIT-30158 (01/2007)

[8] Standard Guide for Computed Tomography (CT) Imaging, ASTM E1441- 11.

[9] Standard Practice for Manufacturing Characterization of Digital Detector Arrays, ASTM E 2597-07, 2007.

[10] Standard Practice for Radiographic Examination, ASTM E 1742-06, 2005. 


\title{
Modeling the X-Ray Process, and X-ray Flaw Size Parameter for POD Studies
}

\author{
Ajay M. Koshti, D.Sc., PE \\ NASA Johnson Space Center \\ March 2014
}




\section{Background and Objective}

- Background

- NDE technique capability is determined by a statistical flaw detection study called Probability of Detection (POD) analysis.

- In many instances the capability is given as a function of a flaw size parameter.

- The discontinuity is usually considered to be a crack or behaving like a crack in terms of affecting the structural integrity of the material.

- Examples of flaw size parameter

- Surface length of the crack is used as the flaw size parameter in Dye Penetrant and Magnetic Particle Testing.

- Area of crack face correlates to the capability of Ultrasonic Testing.

- Flaw depth-to-part-thickness ratio (a/t) is used as the flaw size parameter for X-ray inspection by NASA.

- The flaw size parameter is based on the flaw detection physics of the NDE method.

- A monotonically increasing signal response is expected with increasing flaw size parameter in the desired range of the parameter.

- Basis for a/t ratio as the X-ray flaw size parameter is based on the fact that increasing feature depth in path of the X-ray beam increases the film contrast of the feature.

- Objective of This Work

- Develop a more advanced X-ray (flaw size) parameter with goal of detecting smaller flaws reliably.

- Calculate the $X$-ray parameters for varying cases to assess its applicability. 


\section{Signal Response POD Analysis per MIL-HDBK-1823}

Plot of Experimental Data with $90 \%$ Data Bounds, Linear Model with $95 \%$ Confidence Bounds, and Noise Distribution

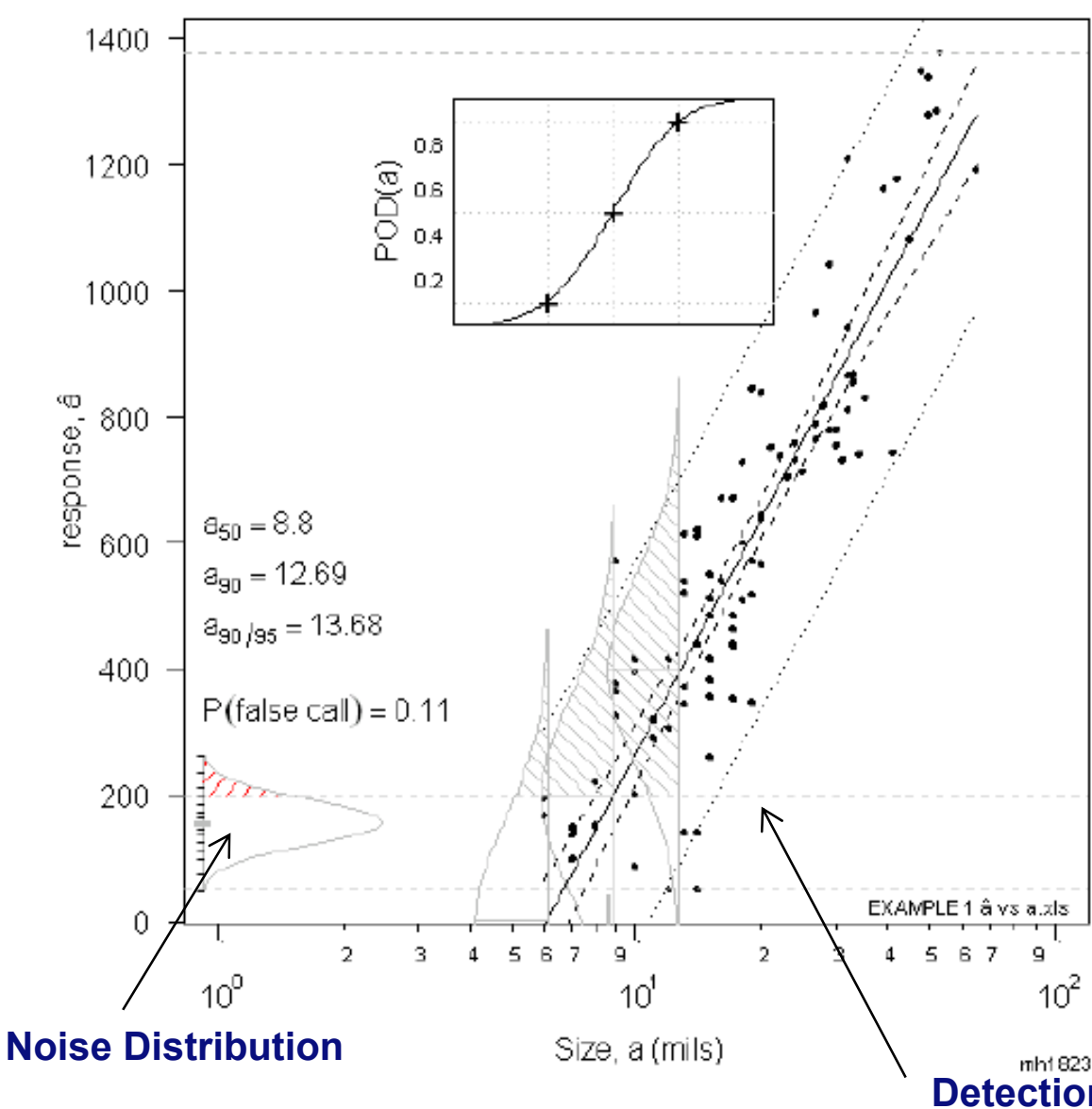

Detection Threshold

Fig. 1: POD Analysis Example from MIL-HDBK-1823A ${ }^{1}$
POD Curve with 95\% Confidence Bounds.

$200 / 95<$ Reliably detected Flaw size parameter

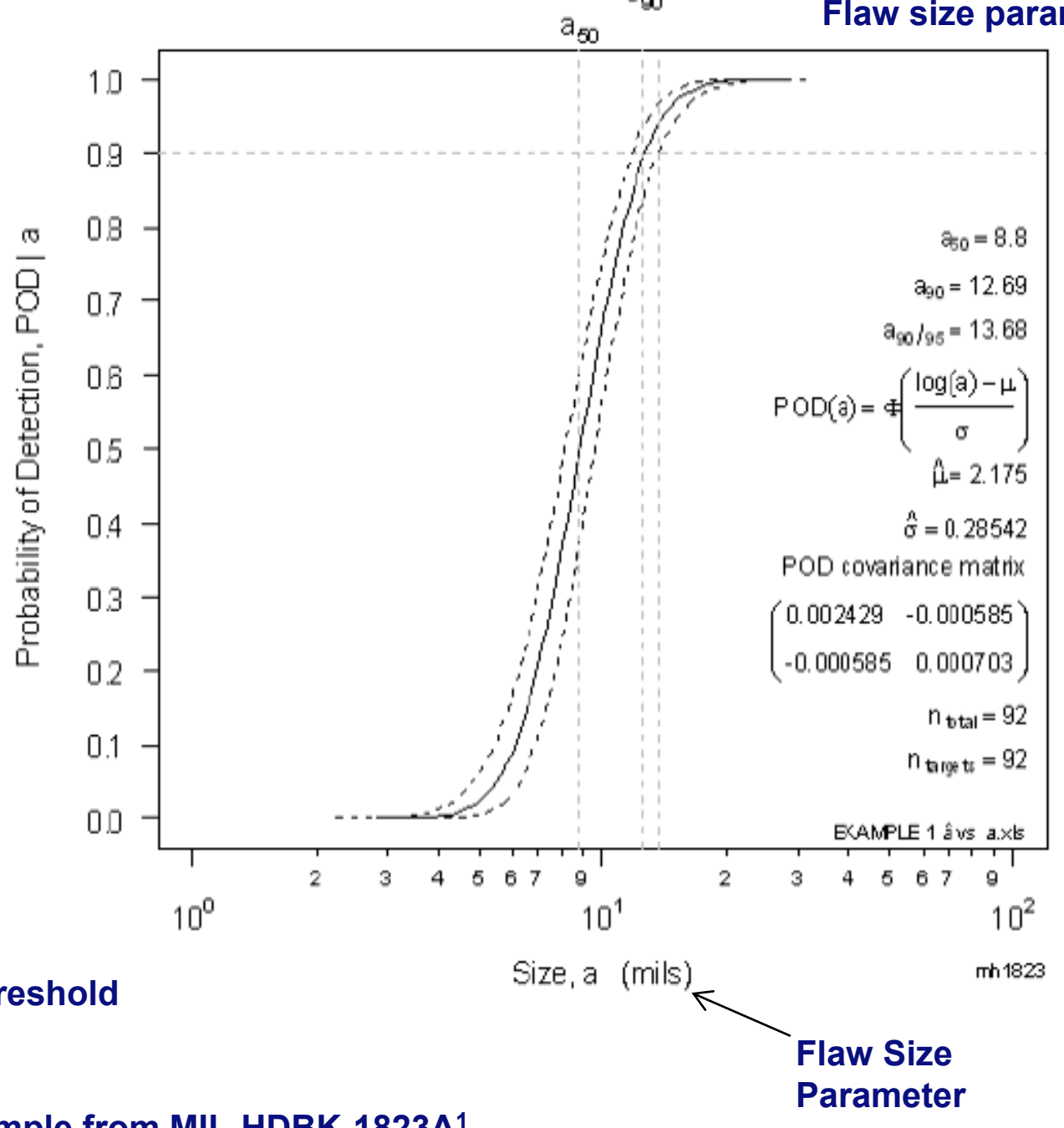

Reference 1: MIL-HDBK-1823A, Nondestructive Evaluation System Reliability Assessment, April 7, 2009. 

Detector

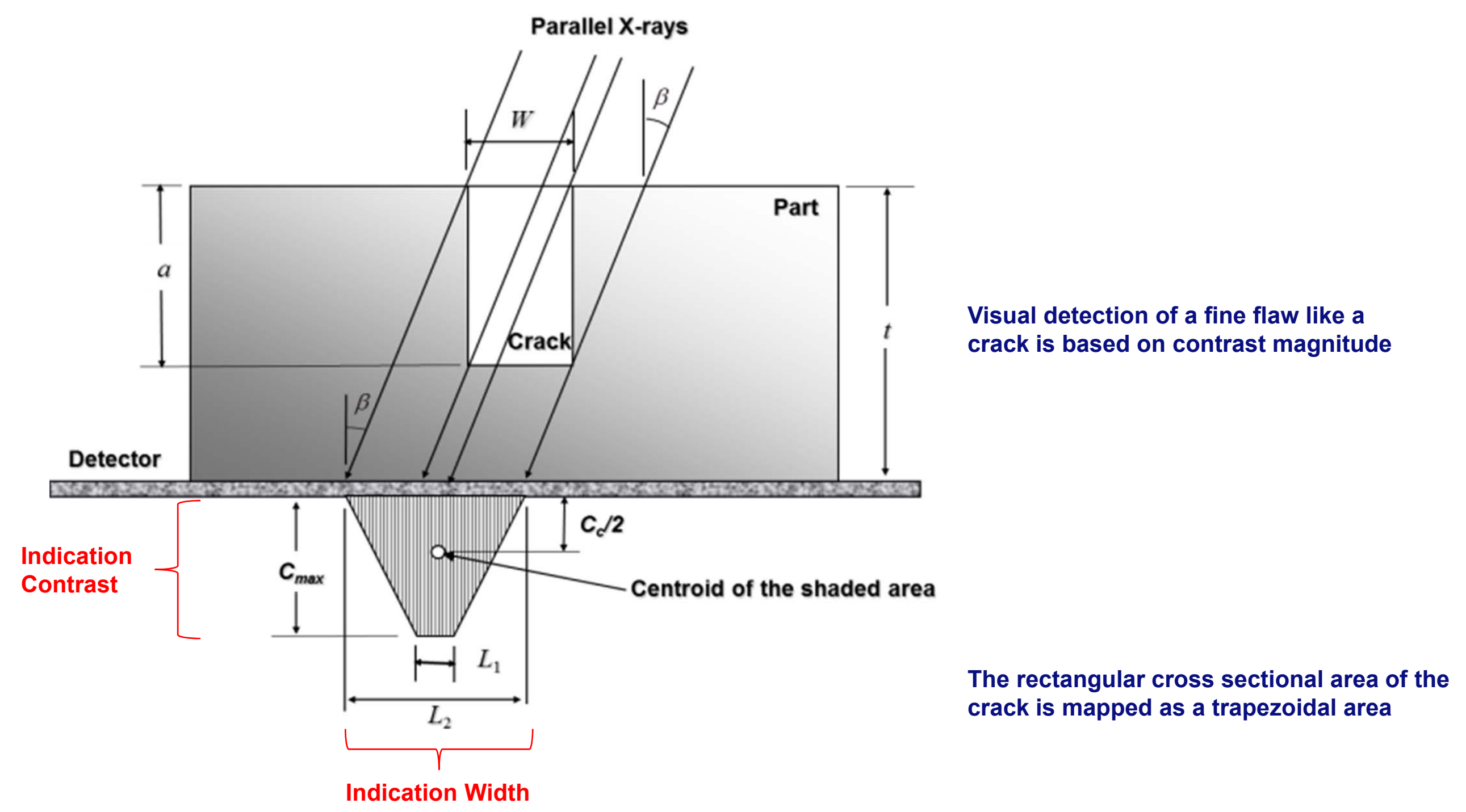

Fig. 2: Cross Sectional Geometry of Part, Slot, and X-ray Shadow Profile on the Detector 


\section{Issue with Flaw Size Parameter a/t}

- Currently, for film radiography crack detection,

- $a / t_{90 / 95}=70 \%$ for Standard NDE

- Requirements: ASTM 1742, minimum radiographic inspection sensitivity level shall be 2$1 \mathrm{~T}$, film density shall be 2.5 to 4.0 , and center axis of the radiation beam shall be within $+/$ 5 degrees of the assumed crack plane orientation.

- Not specific to X-ray procedure, application and operator. No 90/95 demonstration is needed.

$-a / t_{90 / 95}=60 \%$ for Special NDE

- The Special NDE techniques also control technique parameters.

- Special NDE is specific to a given X-ray procedure and operator. A 90/95 demonstration is needed.

- Existing data ${ }^{2}$ shows that $a / t_{90 / 95}=20 \%$ can be achieved on Aluminum fatigue crack specimens.

- Work by Frank Sugg ${ }^{2}$ suggests that lowering $a / t_{90 / 95}$ to $20-40 \%$ is practical.

- There is a need to qualify smaller $a / t_{90 / 95}$ flaw size to improve utility of X-ray NDE in some applications.

- An approach would be to account for most of the technique set-up factors analytically in a model, control remaining factors and perform POD qualification testing.

- This paper develops an analytical model called the "X-ray parameter" which combines the flaw size and set-up parameters, signal response calibration and detector resolution in a simulated or pseudo image contrast number. Flaw size dimensions are some of the many input quantities constituting the X-ray parameter.

- The X-ray parameter may help in implementing special X-ray procedures with lower values of $a / t_{90 / 95}$

- Reference 2: Improved Radiographic Inspection capability for Detecting flaws, Report \# SD 76-SH0196, F. E. Sugg, Rockwell International, 1976 


\section{Considerations Towards the New X-ray Parameter}

- The new X-ray parameter incorporates

1. The flaw size including flaw width

2. Set-up parameters: radiation angle with respect to crack plane (normal and parallel components), source size and source-part-detector distances (geometric unsharpness and magnification), detector angle (image distortion),

3. Signal response calibration (kV, film type, screens, filters, single wall versus double wall and other X-ray technique related parameters), noise (for POD analysis)

4. Detector resolution (Modulation Transfer Function)

to give simulated or pseudo image contrast.

- The new parameter should correlate to more technique and flaw features as dictated by the first principles.

- Material factors are not modeled and are assumed to be controlled

- Weld and parent material alloys, dressed versus undressed welds, presence of conditions that increase spatial noise (grain structure and scattering).

- Simple part geometry is assumed

- $\quad$ The method should be validated by comparing the results to experimental work including POD demonstrations.

- Comparing results to simulations using the commercial software would also help in the validation.

- A possible outcome of this approach would be qualification of the X-ray parameter $\boldsymbol{P}_{90 / 95}$ value with a set of requirements including requirements on material, its form, and technique as well as lower limit on a/t to represent worst cases in the POD demonstration.

- A smaller a/ $t_{90 / 95}$ (e.g. 20-30\%) may be extracted from the qualified $P_{90 / 95}$ in a given technique. 


\section{Flow Chart of the Model Development}

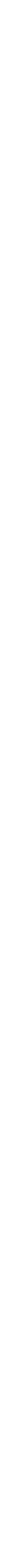

Fig. 3: Flow Chart of the Model Development

Ajay M. Koshti

SPIE NDE \& Smart Structures,

March 2014 


\section{X-ray parameter in Model 1 with Parallel Rays}

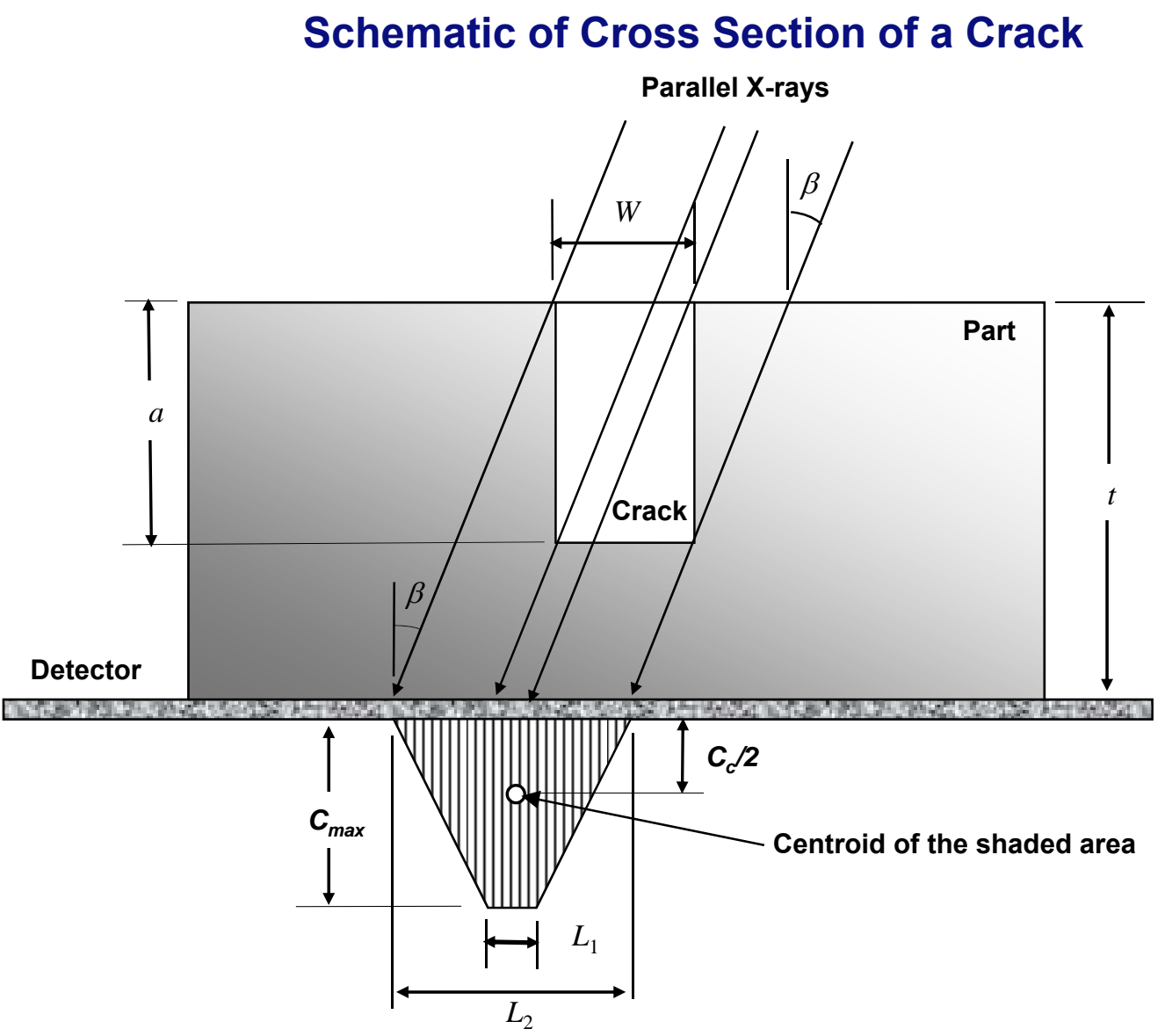

- Above image shows normal component of the X-ray beam angle

- Vertical hatched shaded area indicates relative change in thickness in path-length of X-ray

- X-ray parameter and the equivalent indication width are used in computing the simulated contrast.

$$
P_{c, x} L_{e, x}=a W / t \quad x=\text { model number } 1,2,3 \text { or } 4
$$

\section{Assumptions : Image density} proportional to ray length in material

\section{Parallel rays in the plane of figure}

$$
\begin{aligned}
& \text { Inner } \quad L_{1,1}=|W-a \tan \beta| \\
& \text { Width } \\
& \text { Outer } \\
& \text { Width } \\
& \begin{array}{l}
\text { Equivalent } \\
\text { Indication } \\
\text { Width }
\end{array}
\end{aligned}
$$

Mode 1: $W \geq a \tan \beta$

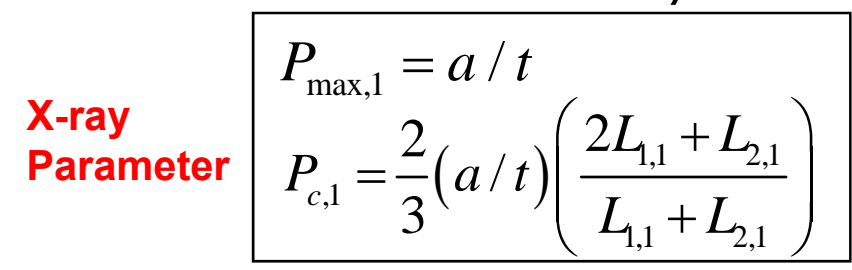

Mode 2: $W \leq a \tan \beta$

X-ray

Parameter

$P_{\max , 1}=\frac{W}{t \tan \beta}$
$P_{c, 1}=\frac{2}{3}\left(\frac{W}{t \tan \beta}\right) \frac{2 L_{1,1}+L_{2,1}}{L_{1,1}+L_{2,1}}$

- Model 1 is used to derive models 2 and 3. 


\section{Model 2 with Unsharpness}

- Geometric unsharpness increases the indication size and reduces film contrast

- Assume that the film is normal to the X-rays within +/-6 degree

- Assume that the parallel component of beam angle is within +/-6 degree

Inner Width

$L_{1,2}=|a \tan \beta-W|\left(\frac{d_{1}+d_{2}}{d_{1}}\right)-U_{n} \mid$

Mode $1 \mathrm{X}$-ray Parameter

$P_{c, 2}=\frac{2}{3}\left(\frac{a}{t}\right)\left(\frac{2 L_{1,2}+L_{2,2}}{L_{1,2}+L_{2,2}}\right)\left(\frac{L_{1,1}+L_{2,1}}{L_{1,2}+L_{2,2}}\right)$
Equivalent Indication Width

$$
L_{e, 2}=\frac{a W}{t} \frac{1}{P_{c, 2}}
$$

Outer Width

$L_{2,2}=(a \tan \beta+W)\left(\frac{d_{1}+d_{2}}{d_{1}}\right)+U_{n}$
Mode 2 X-ray Parameter

$P_{c, 2}=\frac{2}{3}\left(\frac{W}{t \tan \beta}\right)\left(\frac{2 L_{1,2}+L_{2,2}}{L_{1,2}+L_{2,2}}\right)\left(\frac{L_{1,1}+L_{2,1}}{L_{1,2}+L_{2,2}}\right)$

- $U_{\mathrm{n}}=U_{\mathrm{g}}+U_{\text {other }}$

- $U_{\text {other }}$ represents other unsharpness effects due to rippled surface of the weld, scattering, weld grain structure, film grain etc.

- Estimation of $U_{\text {other }}$ will require comparison of calculated $L_{1}$ and $L_{2}$ with measured values of the same using a high power microscope and/or film digitizer. 

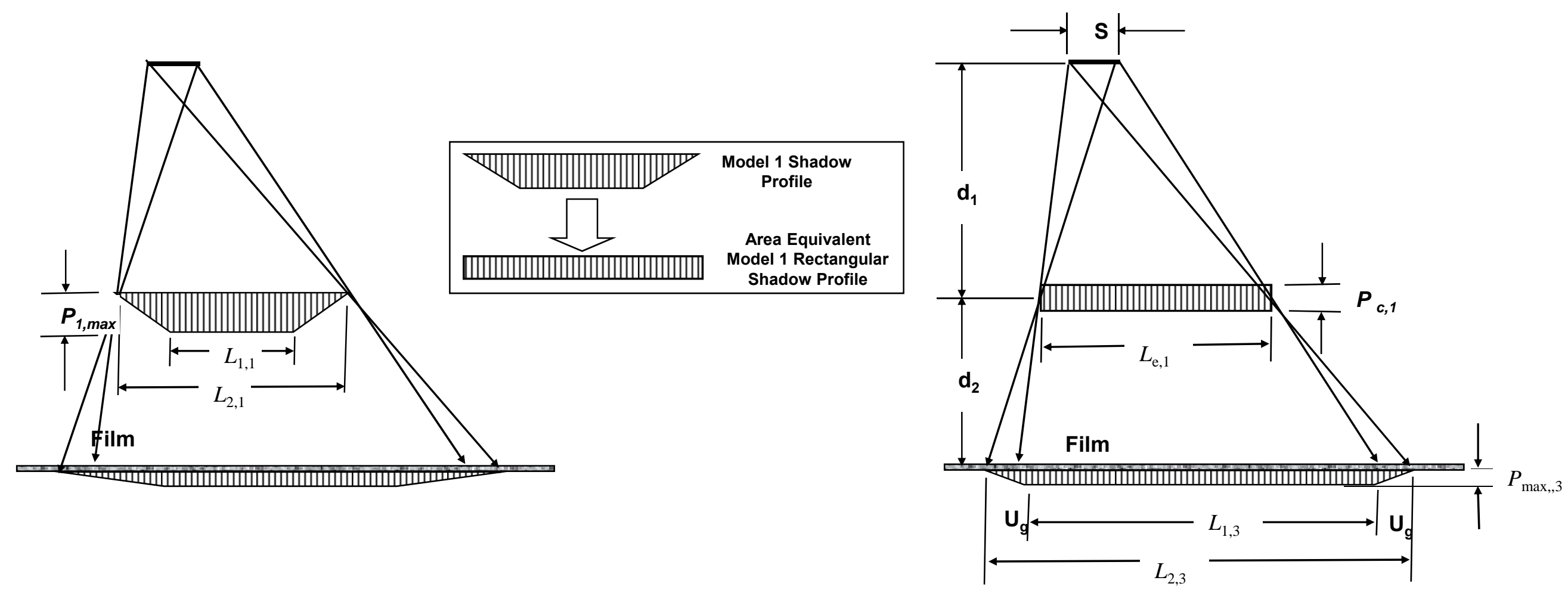

Fig. 4: Incorporating Geometric Unsharpness in Model 3 


\section{Model 3 with Geometric Unsharpness}

\section{Geometric Unsharpness}

$$
U_{g}=\frac{S d_{2}}{d_{1}}
$$

Inner Width

$L_{1,3}=\left|\frac{L_{x, 3}\left(d_{1}+d_{2}\right)}{d_{1}}-U_{g}\right|$

Outer Width

$$
L_{2,3}=\frac{L_{x, 3}\left(d_{1}+d_{2}\right)}{d_{1}}+U_{g}
$$

Equivalent Indication Width

$$
L_{e, 3}=\frac{a W}{t} \frac{1}{P_{c, 3}}
$$

Mode 1 and 2 X-ray Parameter

$$
P_{c, 3}=\frac{2}{3} P_{\max , 3} \frac{2 L_{1,3}+L_{2,3}}{L_{1,3}+L_{2,3}}
$$

Where, $\quad L_{x, 3}=\frac{3}{4} \frac{\left(L_{1,1}+L_{2,1}\right)^{2}}{\left(2 L_{1,1}+L_{2,1}\right)}$

Where, $\quad P_{\max , 3}=P_{\max , 1} \frac{L_{1,1}+L_{2,1}}{L_{1,3}+L_{2,3}}$ 


\section{Calibrating Detector Response Function $f_{\mathrm{n}}$ to Step Wedge a/t}

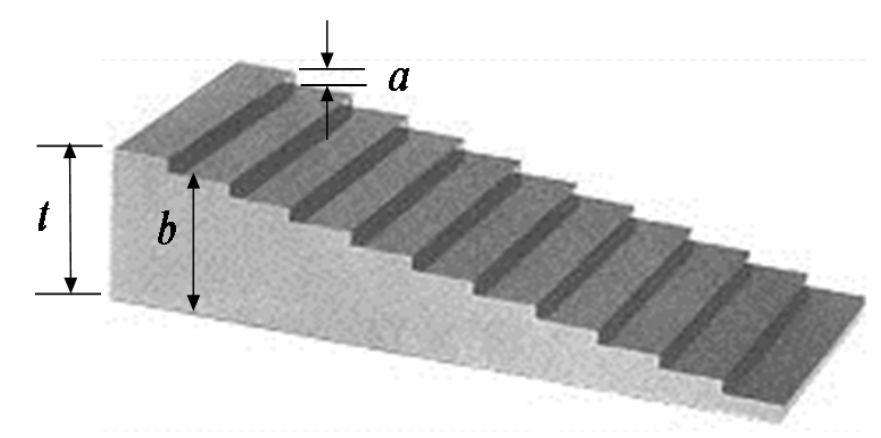

Fig. 5: An Example of An Aluminum Step Wedge

Modulation Accounted X-ray parameter

$$
P_{c, M}=g_{n}\left(1 / L_{l p}\right) P_{c}
$$

Normalized Contrast

$$
C_{f\left(P_{c}\right), M}=M_{n} \frac{f_{n}\left(P_{c}\right)}{f_{n}(1)}=g_{n}\left(L_{l p}\right) \frac{f_{n}\left(P_{c}\right)}{f_{n}(1)}
$$

Image Contrast

$$
S_{f\left(P_{c}\right), M}=M_{n} f_{n}\left(P_{c}\right)=g_{n}\left(1 / L_{l p}\right) f_{n}\left(P_{c}\right)
$$

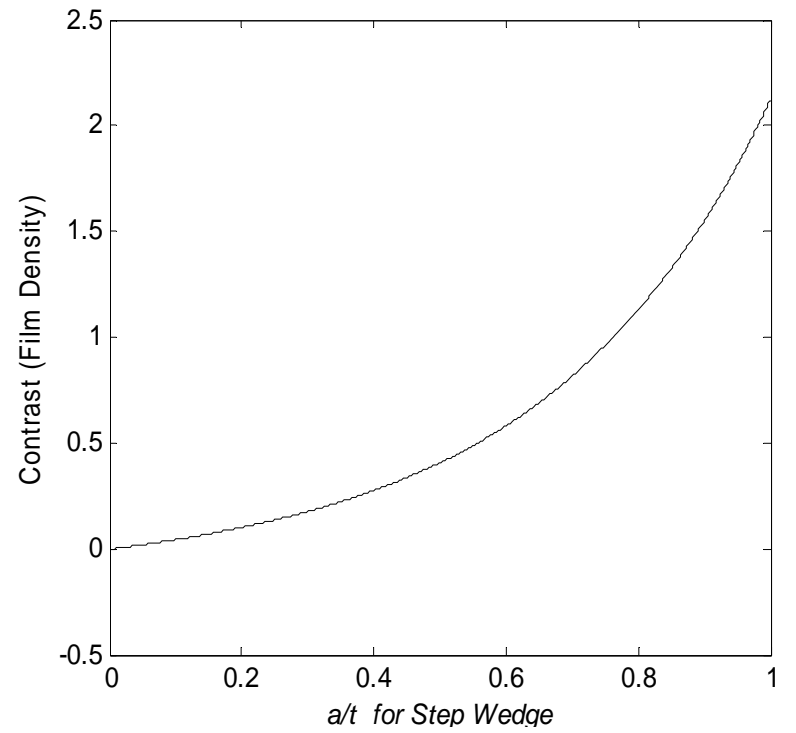

Fig. 7: Detector Response Function for a Film

$X$-ray parameter $P_{c}$ is used in place of a/t when using the calibration curves to calculate simulated contrast.

Equivalent Indication Width, $L_{e}$ is used in place of $L_{1 p}$ when applying function $g_{\mathrm{n}}$ to calculate simulated contrast 


\section{Modulation Transfer Function, $g_{\mathrm{n}}$}

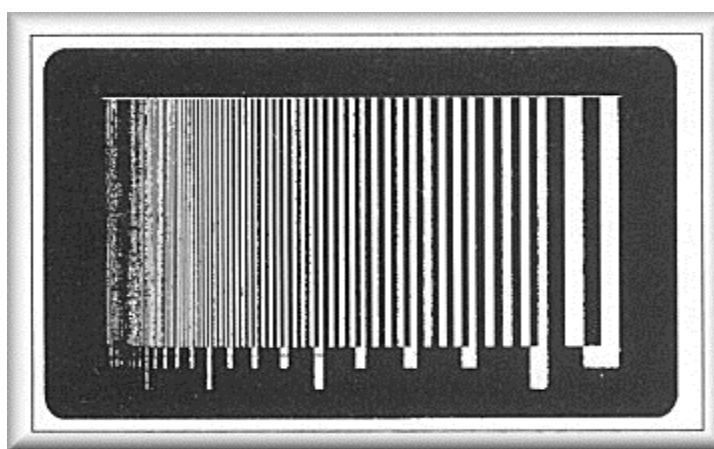

Fig. 8: A Line Pair Resolution Target Made from a $0.05 \mathrm{~mm}$ thick lead shim
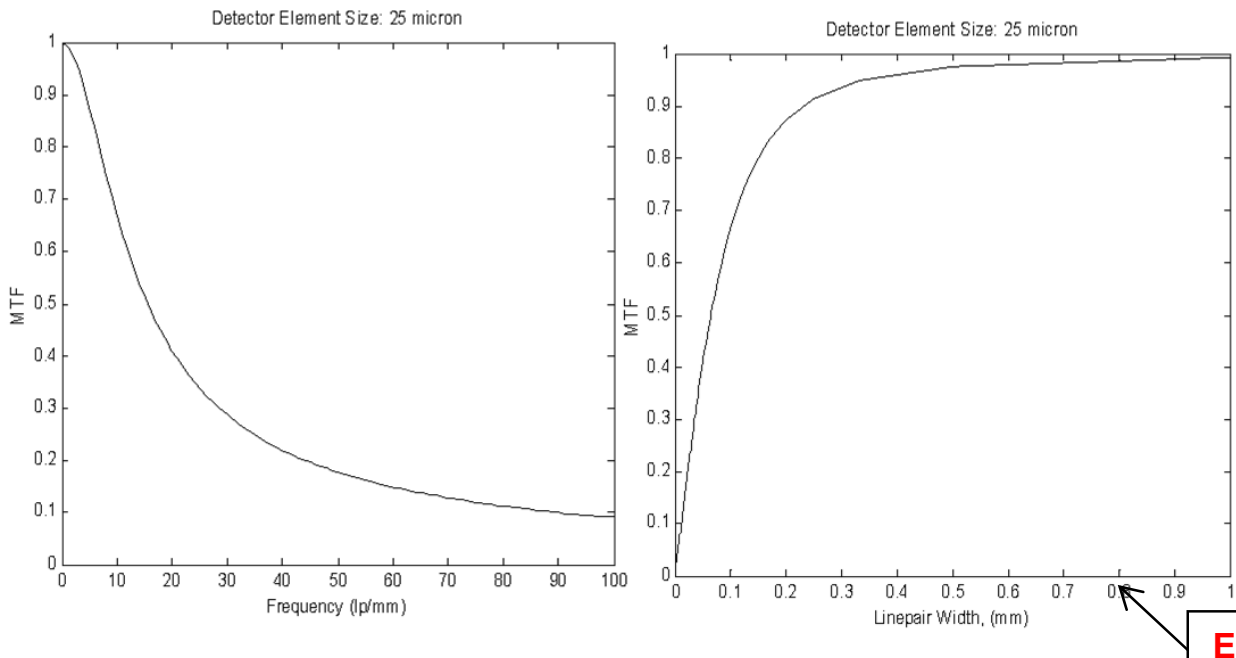

Fig. 9: MTF Used for the Film in the Simulation
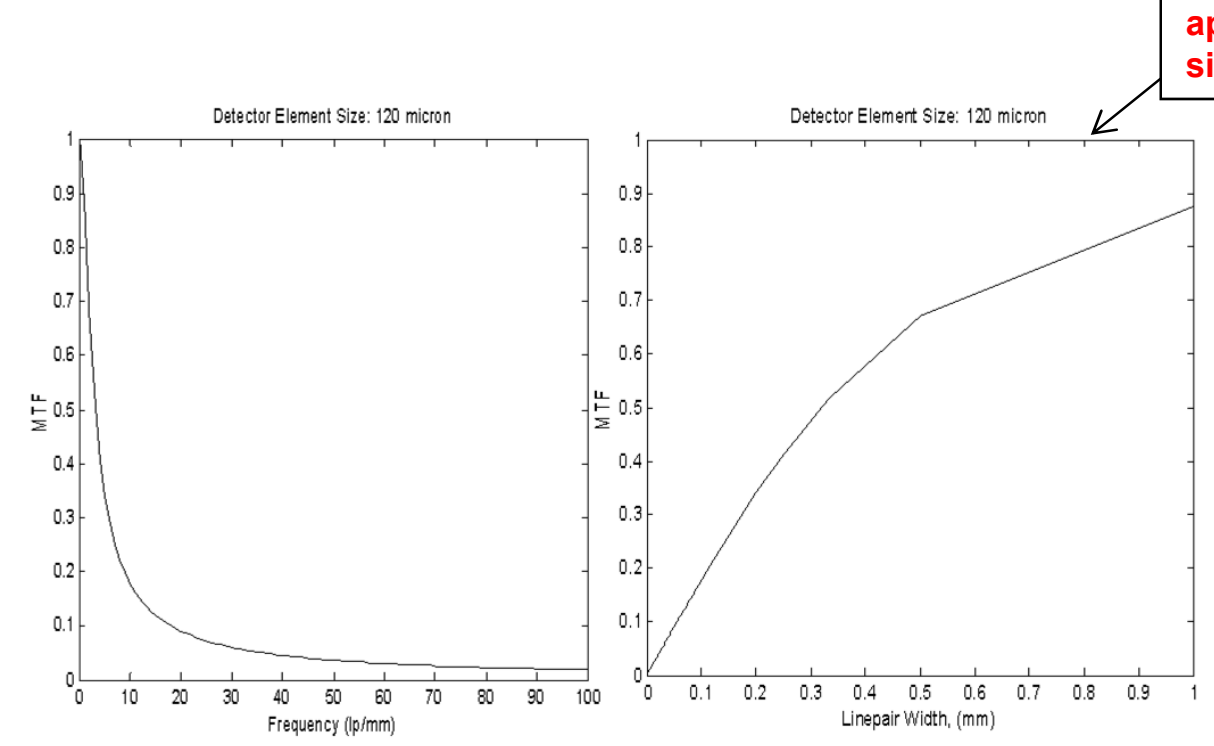

Fig. 10: MTF Used for a Digital Detector in the Simulation
Equivalent Indication Width, $L_{e}$ is used in place of $L_{1 p}$ when applying function $g_{n}$ to calculate simulated contrast 


\section{X-ray Parameter $\boldsymbol{P}_{\mathrm{c}, 3}$ Calculation Example}

Source to part distance $=60 \mathrm{~cm}$

Part to detector distance $=10 \mathrm{~cm}$

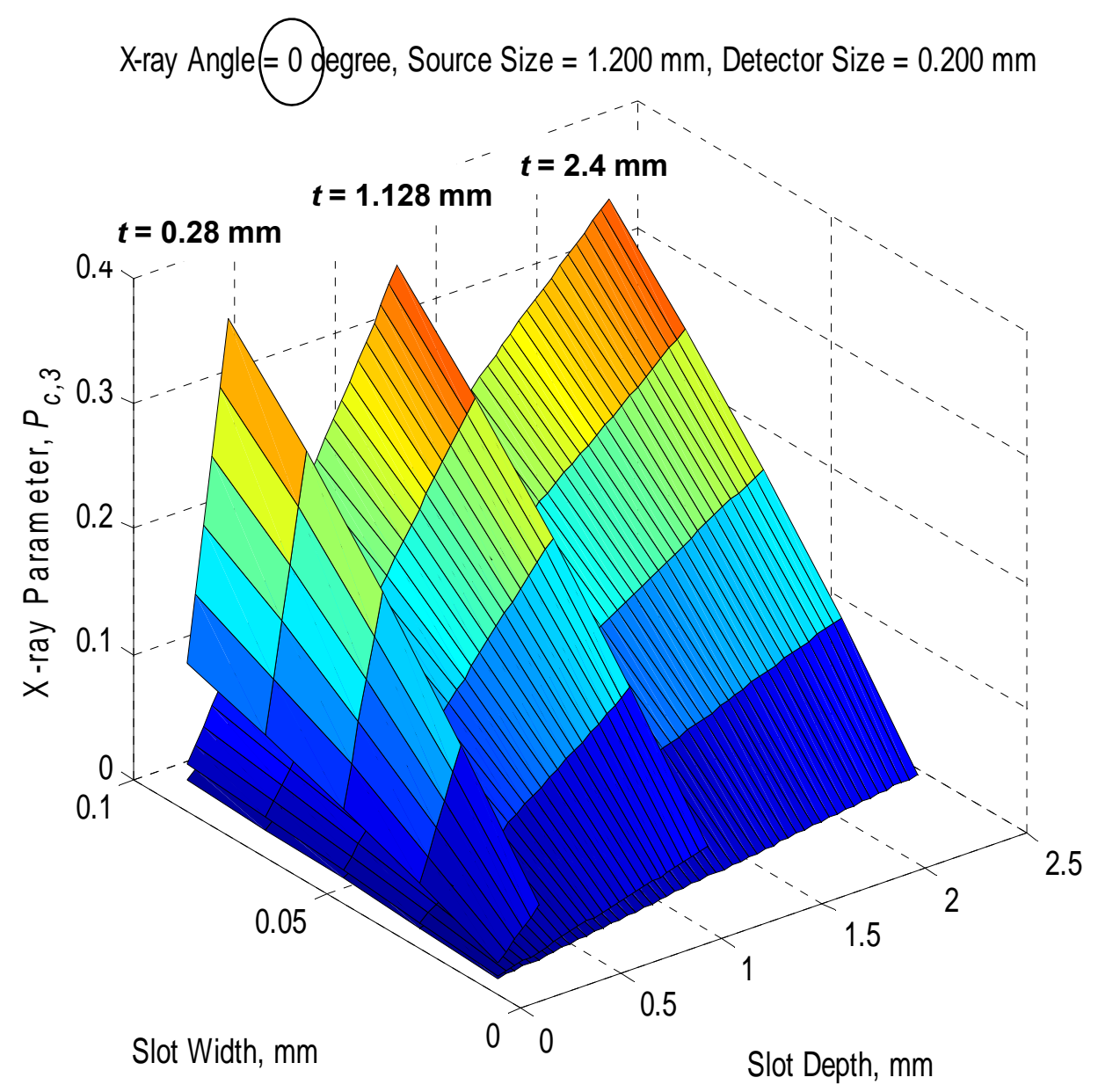

Fig. 11: X-ray parameter, source $=1.2 \mathrm{~mm}$

X-ray parameter $P_{c}$ increases with slot depth and width.

$X$-ray parameter $P_{c}$ for a given a/t is same for varying plate thicknesses for X-ray angle of 0 degree to part normal. 


\section{X-ray Parameter $\boldsymbol{P}_{\mathrm{c}, 3}$ Calculation Example}

Source to part distance $=60 \mathrm{~cm}$ Part to detector distance $=10 \mathrm{~cm}$

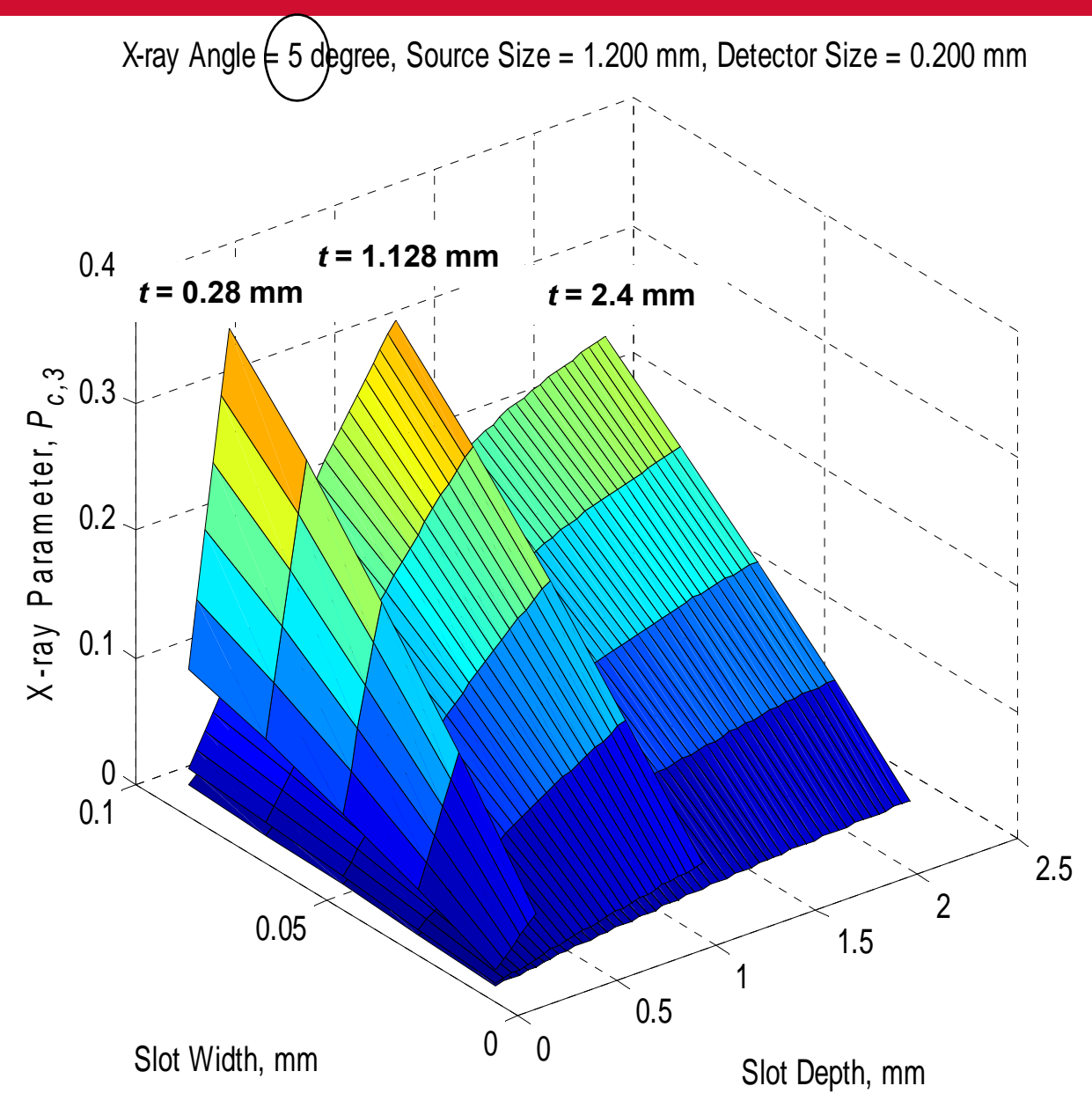

Fig. 12: X-ray parameter, source $=1.2 \mathrm{~mm}$

X-ray angle is different between simulation data of Fig. 11 (angle = 0) and Fig. 12 (angle = 5 deg.). Higher X-ray angle reduces the X-ray parameter. The effect is higher for thicker parts. 


\section{X-ray Parameter $\boldsymbol{P}_{\mathrm{c}, 3}$ Calculation Example}

X-ray Angle $=0$ degree, Source Size $=0.200 \mathrm{~mm}$, Detector Size $=0.200 \mathrm{~mm}$

Source to part distance $=60 \mathrm{~cm}$ Part to detector distance $=10 \mathrm{~cm}$

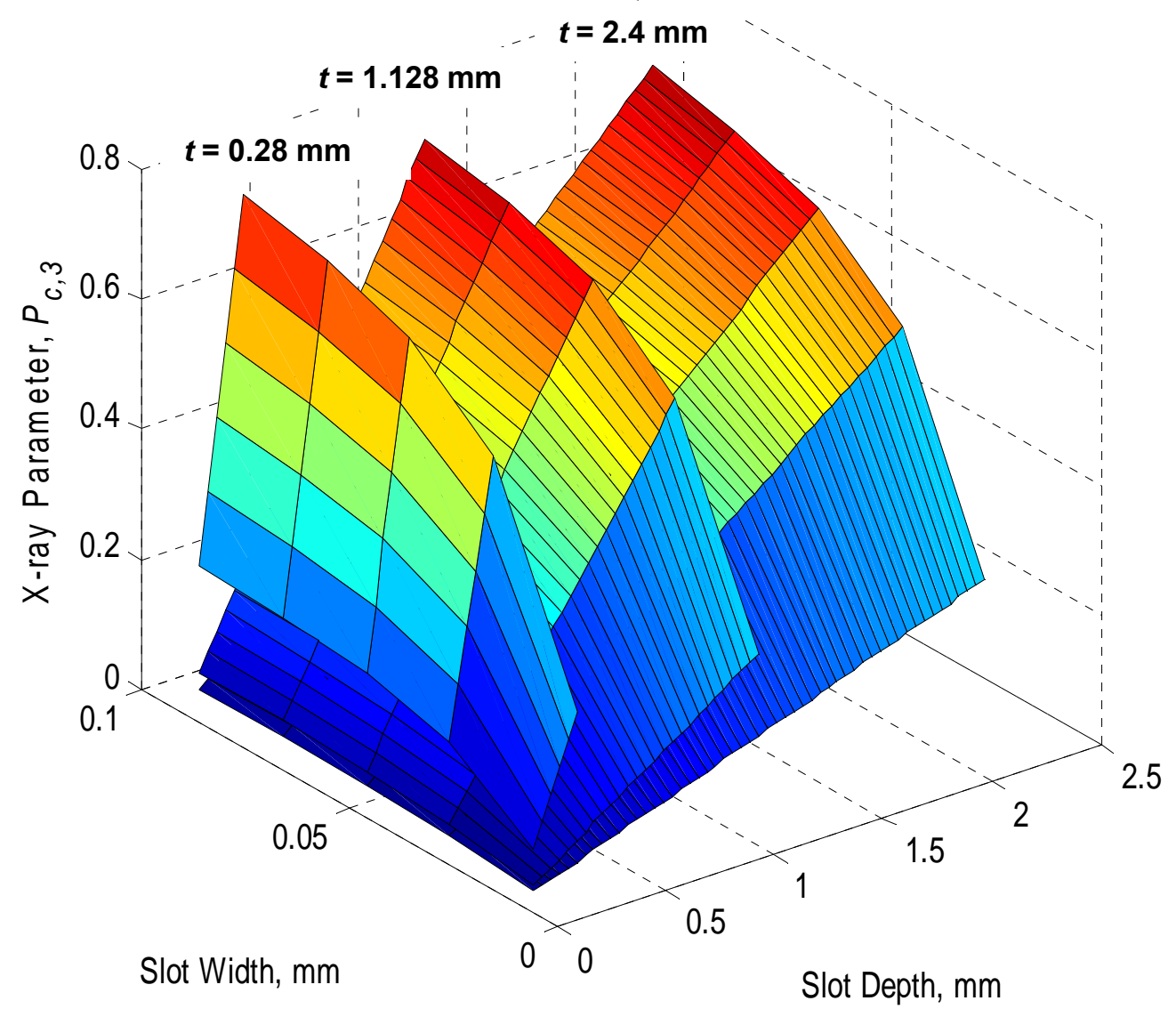

Fig. 13: X-ray parameter, source $=0.2 \mathrm{~mm}$

Source size is different between Simulation Data of Fig. 11 (Source Size $=1.200 \mathrm{~mm}$ ) and Fig. 13 (Source Size = 0.200 mm). Smaller source size in Fig. 13 gives higher values of the flaw size parameter. 


\section{X-ray Parameter $\boldsymbol{P}_{\mathrm{c}, 3, \mathrm{M}}$ Calculation Example}

Source to part distance $=60 \mathrm{~cm}$ Part to detector distance $=10 \mathrm{~cm}$

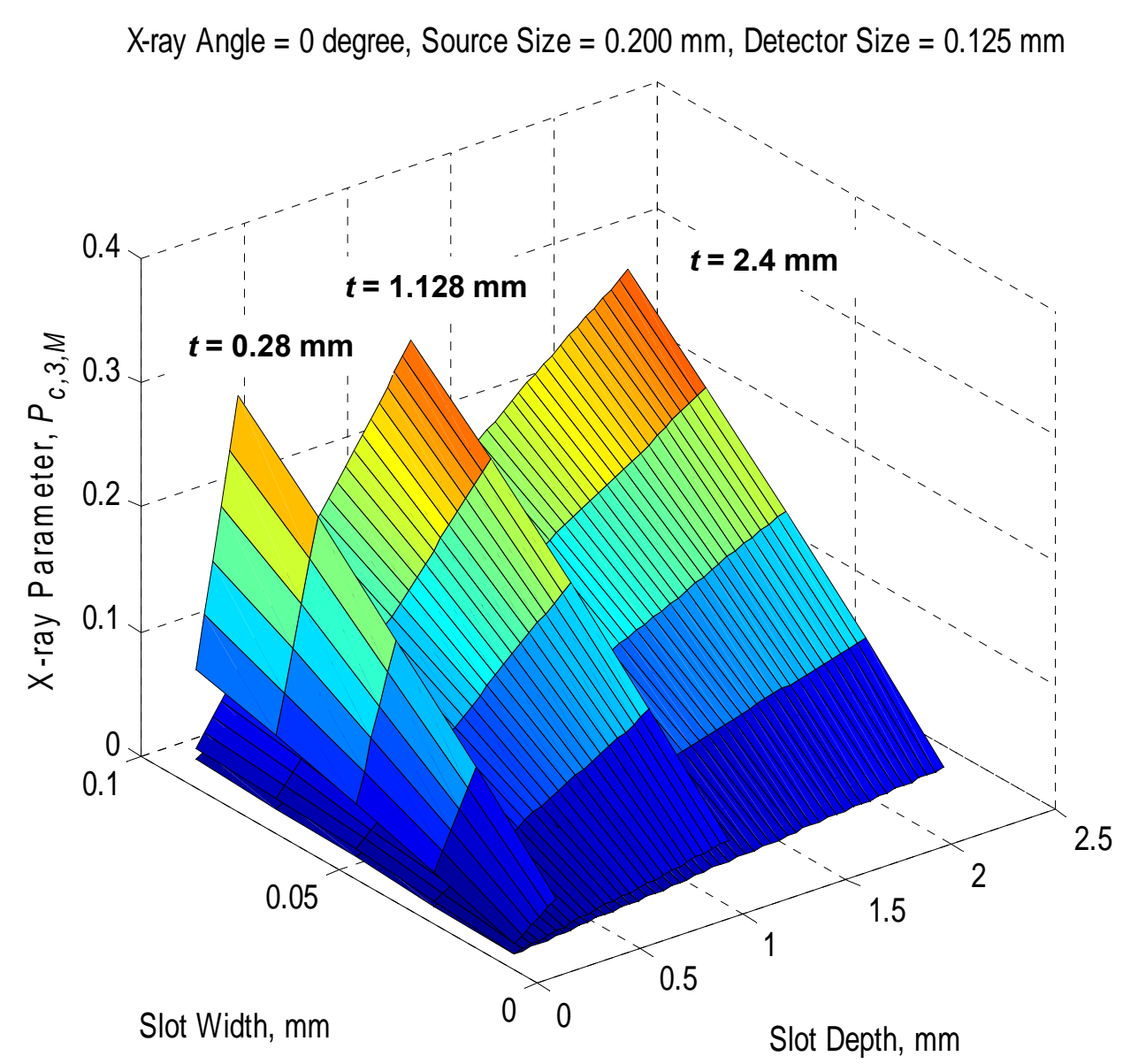

Fig. 14: X-ray parameter, source $=0.2 \mathrm{~mm}$, detector resolution $4 \mathrm{Ip} / \mathrm{mm}$ 


\section{Contrast $S_{p c, 3, M}$ Calculation Example}

Source to part distance $=60 \mathrm{~cm}$

Part to detector distance $=10 \mathrm{~cm}$

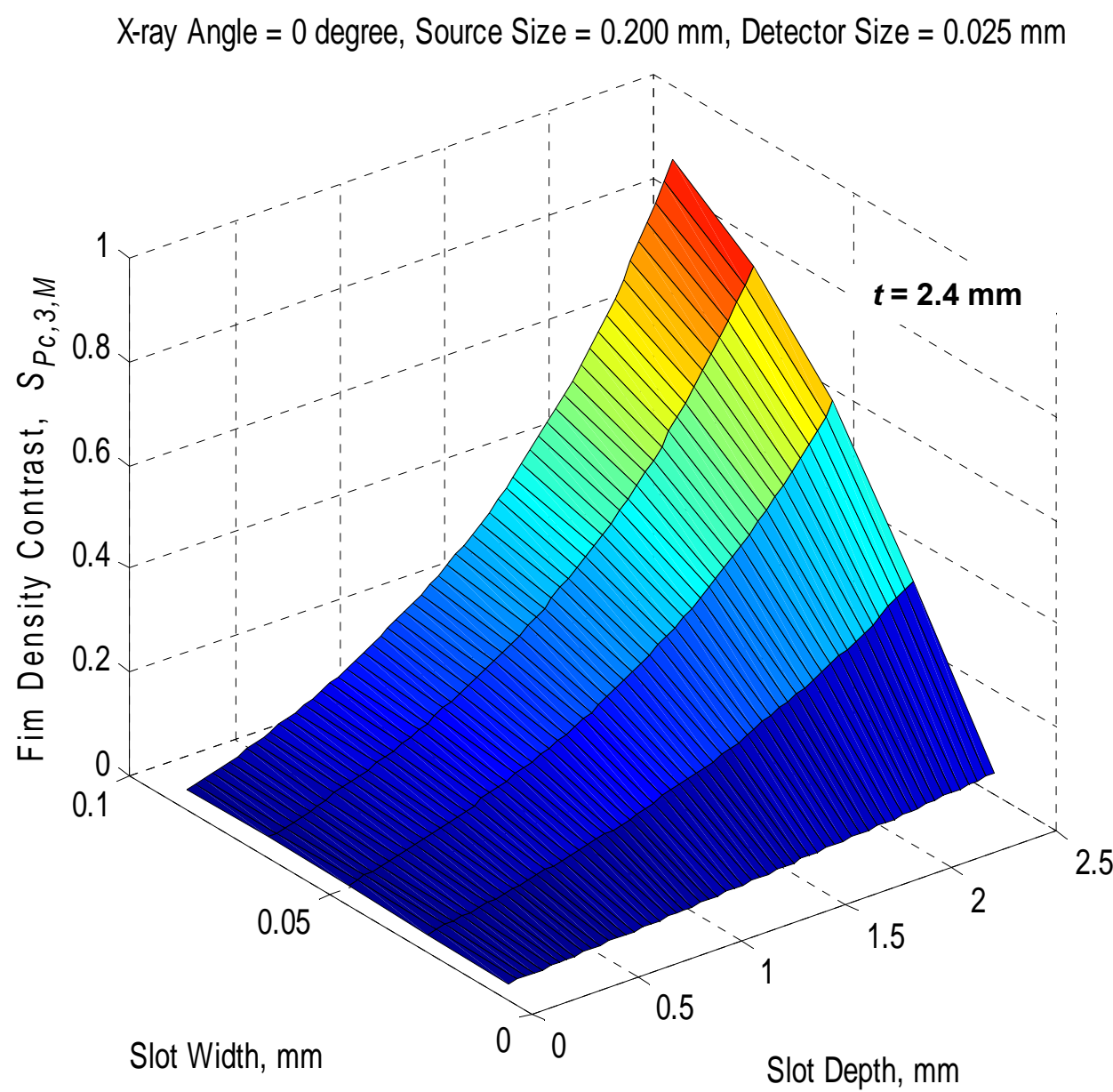

Uses calibration curve of Fig. 7

Fig. 15: Simulated equivalent contrast for film, detector resolution $20 \mathrm{lp} / \mathrm{mm}$, source size $0.2 \mathrm{~mm}$ 


\section{Normalized Contrast $C_{p \mathrm{c}, 3, \mathrm{M}}$ Calculation Example}

Source to part distance $=60 \mathrm{~cm}$ Part to detector distance $=10 \mathrm{~cm}$
X-ray Angle $=0$ degree, Source Size $=0.200 \mathrm{~mm}$, Detector Size $=0.125 \mathrm{~mm}$

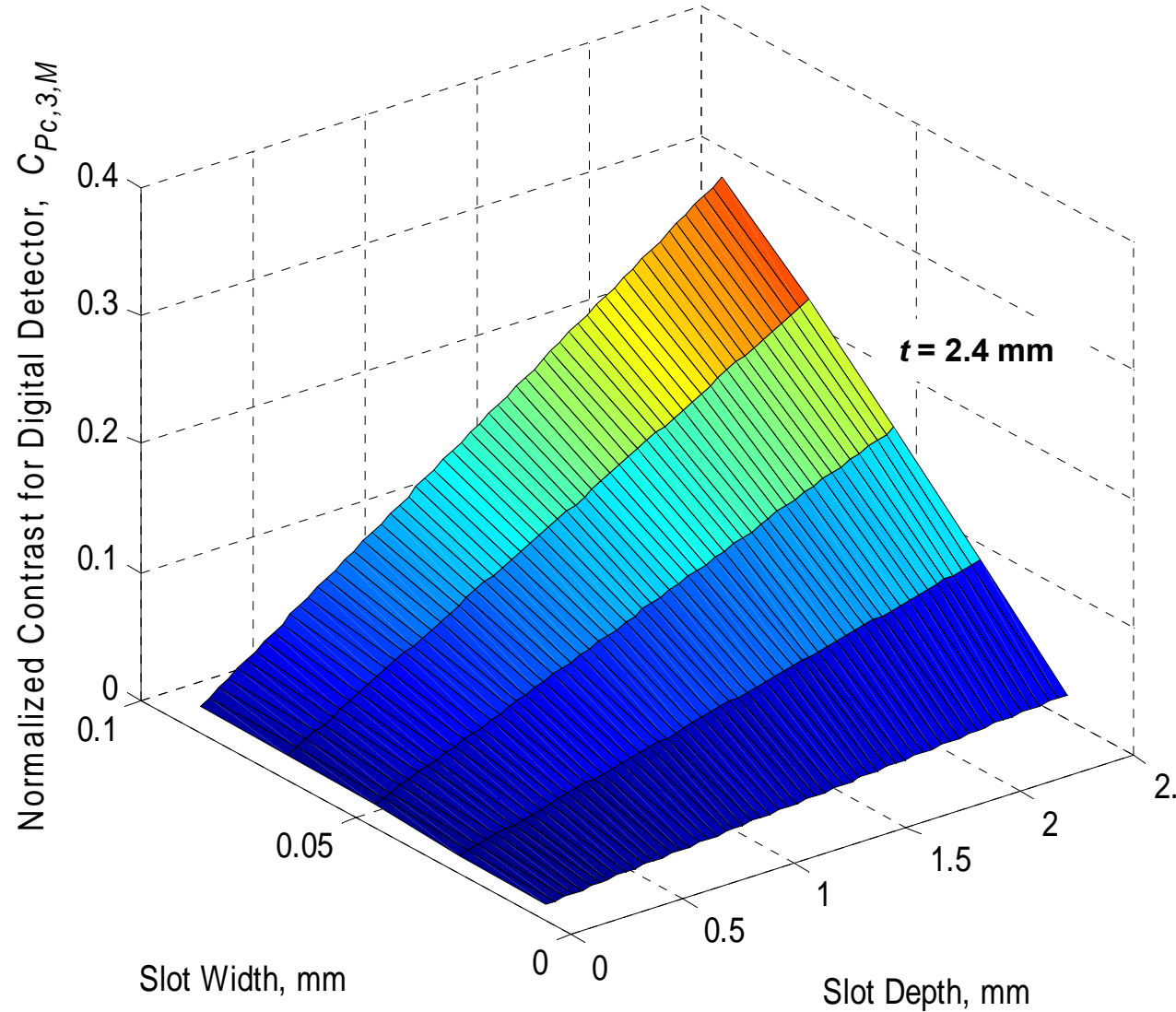

Uses calibration curve of Fig. 6

Fig. 13: Simulated equivalent normalized contrast for a digital detector with resolution $4 \mathrm{Ip} / \mathrm{mm}$ 


\section{Calculations for a $70 \%$ slot and a $2-2 \mathrm{~T}$ Square Hole}

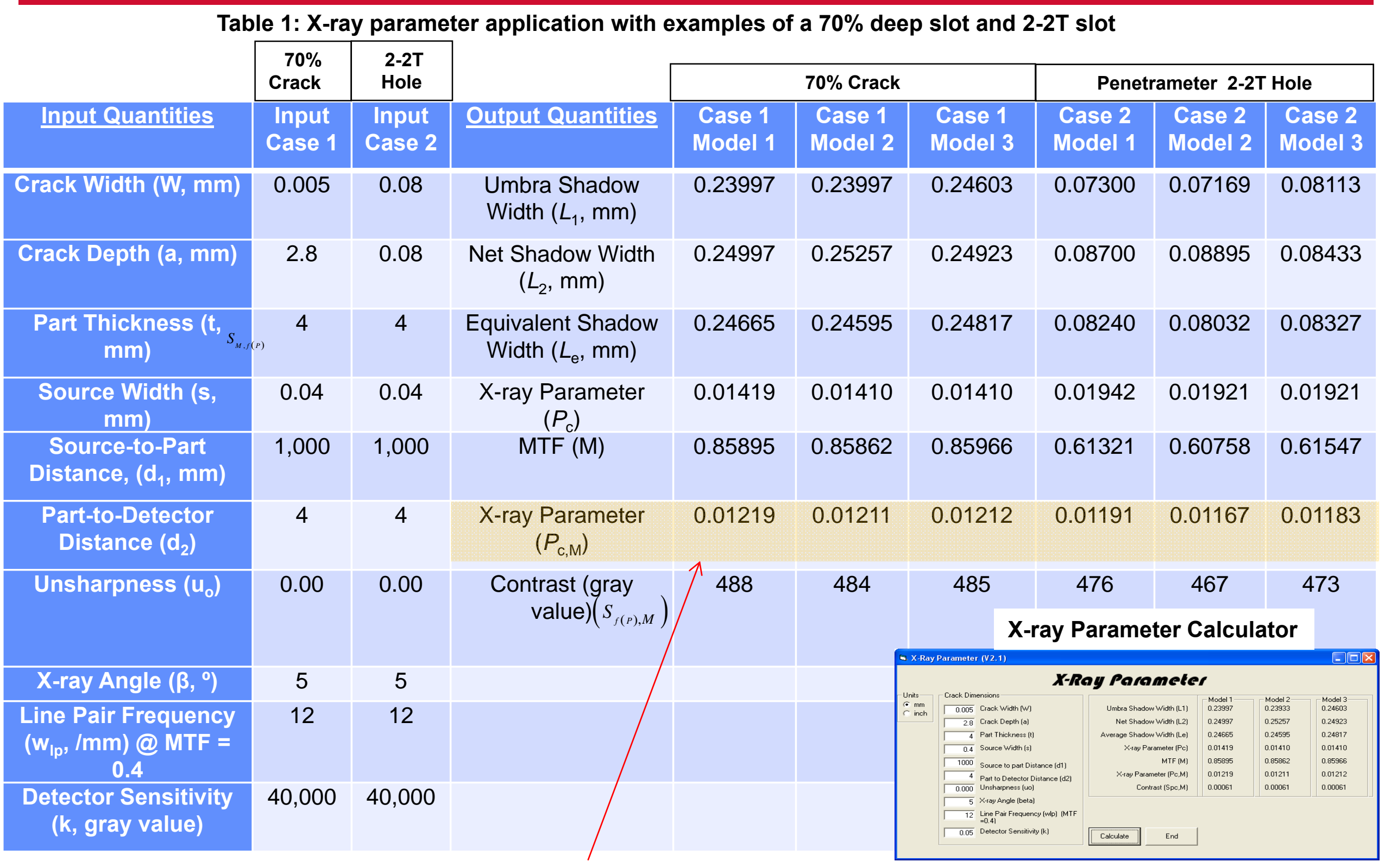

Flaw size parameter values are approximately same between the $2-2 \mathrm{~T}$ hole and $70 \%$ slot.

Ajay M. Koshti

SPIE NDE \& Smart Structures,

March 2014 


\section{Summary}

- The paper provides models for X-ray parameters.

- X-ray parameters are designed to relate to image contrast.

- X-ray parameters defined here are more advanced than the currently used parameter a/t.

- A process of the detector response curve as a function of ratio a/t using a step wedge is used. Detector response curve is used in computation of the X-ray parameters (including the simulated contrast and simulated normalized contrast).

- The modulation transfer function is used in the X-ray parameter models.

- The simulated contrast and simulated normalized contrast can also be used for establishing correlation with actual contrast and flaw detectability.

- If the X-ray parameter $\boldsymbol{P}_{90 / 95}$ is validated for certain type of inspection, the computer calculator application mentioned here, can provide a simple way to evaluate an X-ray technique for reliable detection of cracklike flaws. 


\section{ACKNOWLEDGEMENT}

Following individuals provided valuable technical comments to this work.

- Dr. Daniel Schneberk, Lawrence Livermore National Laboratory;

- Dr. Donald Roth, NASA Glenn Research Center

- David Stanley, NASA Johnson Space Center

- James Walker, NASA Marshall Space Flight Center 